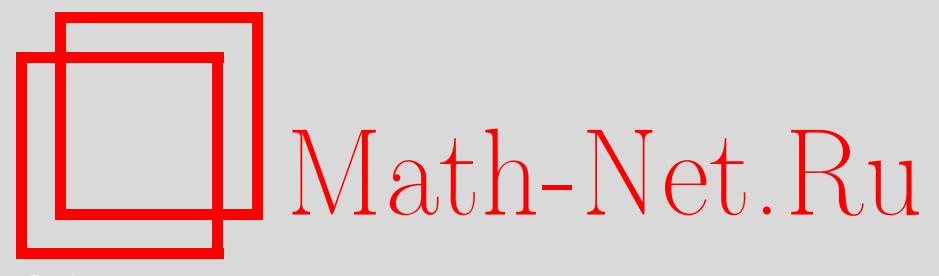

Р. М. Колпаков, О многозначных преобразованиях конечных множеств бинарных распределений с рациональными вероятностями, Дискрет. матем., 2005, том 17, выпуск 1, 102-128

DOI: https://doi.org/10.4213/dm91

Использование Общероссийского математического портала Math-Net.Ru подразумевает, что вы прочитали и согласны с пользовательским соглашением http://www.mathnet.ru/rus/agreement

Параметры загрузки:

IP : 44.207 .124 .84

26 апреля 2023 г., 15:01:12

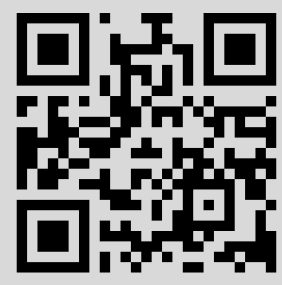




\title{
О многозначных преобразованиях конечных множеств бинарных распределений с рациональными вероятностями
}

\author{
() 2005 г. . М. Колпаков
}

\begin{abstract}
Изучаются дискретные преобразования независимых случайных величин, принимающих конечное число значений с рациональными вероятностями (под дискретным преобразованием случайных величин понимается случайная величина, значение которой является функцией от значений исходных случайных величин). Мы определяем явным образом все возможные вероятностные распределения случайных величин, являющихся дискретными преобразованиями случайных величин с распределениями из произвольного конечного множества бинарных рациональных распределений.

Работа выполнена при поддержке Российского фонда фундаментальных исследований, проект 02-01-00985, и программы Президента Российской Федерации поддержки ведущих научных школ, проект 00-15-96103.
\end{abstract}

\section{1. Введение}

В данной работе мы продолжаем начатые в [4] исследования, касающиеся преобразований бинарных вероятностных распределений с рациональными значениями вероятностей. Такие преобразования играют важную роль в вопросах реализации случайностей, имеющих большое значение для многих областей математической кибернетики (см. $[1,13])$. Под преобразованием вероятностных распределений мы понимаем вероятностное распределение некоторой случайной величины $\zeta_{0}$, значение которой однозначно определяется значениями конечного числа независимых случайных величин $\zeta_{1}, \ldots, \zeta_{k}$ с исходными вероятностными распределениями. Очевидно, что данное преобразование задается функцией $f: \Omega_{1} \times \ldots \times \Omega_{k} \rightarrow \Omega_{0}$, где $\Omega_{i}-$ множество значений случайной величины $\zeta_{i}$, $i=0,1, \ldots, k$. Нами рассматриваются случайные величины, принимающие конечное число значений. Не ограничивая общности, мы можем считать, что такая случайная величина принимает целые неотрицательные значения, а ее вероятностное распределение задается стохастическим вектором, под которым мы понимаем вектор с неотрицательными компонентами, сумма которых равна 1 и $j$-я компонента которого равна вероятности принятия этой случайной величиной значения $j-1$. Пусть вероятностное распределение случайной величины $\zeta_{i}$ задается стохастическим вектором $\mathscr{D}_{i}$, тем самым $\Omega_{i}=\left\{0,1, \ldots, h_{i}-1\right\}$, где $h_{i}$ - размерность вектора $\mathscr{D}_{i}, i=0,1, \ldots, k$. Множество $\Omega_{1} \times \ldots \times \Omega_{k}=\left\{0,1, \ldots, h_{1}-1\right\} \times \ldots \times\left\{0,1, \ldots, h_{k}-1\right\}$ мы будем обозначать через $\Omega\left(\mathscr{D}_{1}, \ldots, \mathscr{D}_{k}\right)$. Для любого подмножества $E$ этого множества обозначим через $\mathbf{P}_{E}\left(\mathscr{D}_{1}, \ldots, \mathscr{D}_{k}\right)$ вероятность того, что набор $\left(\sigma_{1}, \ldots, \sigma_{k}\right)$ значений величин $\zeta_{1}, \ldots, \zeta_{k}$ 
содержится в $E$. Через $\mathscr{D}[j]$ мы будем обозначать $j$-ю компоненту стохастического вектора $\mathscr{D}$. Тогда

$$
\mathbf{P}_{E}\left(\mathscr{D}_{1}, \ldots, \mathscr{D}_{k}\right)=\sum_{\left(\sigma_{1}, \ldots, \sigma_{k}\right) \in E} \mathscr{D}_{1}\left[\sigma_{1}+1\right] \cdot \ldots \cdot \mathscr{D}_{k}\left[\sigma_{k}+1\right],
$$

в случае $E=\varnothing$ мы полагаем сумму (1) равной 0 .

Через $\mathcal{N}_{i}(f)$ обозначим множество всех наборов из $\Omega\left(\mathscr{D}_{1}, \ldots, \mathscr{D}_{k}\right)$, на которых функция $f$ принимает значение $i$. Используя это обозначение, мы определяем компоненты вектора $\mathscr{D}_{0}$ следующим образом:

$$
\mathscr{D}_{0}[j]=\mathbf{P}_{\mathcal{N}_{j-1}(f)}\left(\mathscr{D}_{1}, \ldots, \mathscr{D}_{k}\right), \quad j=1, \ldots, h_{0} .
$$

В дальнейшем мы обозначаем вектор $\mathscr{D}_{0}$ через $\mathbf{P}\left\{f\left(\mathscr{D}_{1}, \ldots, \mathscr{D}_{k}\right)\right\}$. Отметим, что вектор $\mathbf{P}\left\{f\left(\mathscr{D}_{1}, \ldots, \mathscr{D}_{k}\right)\right\}$ определен тогда и только тогда, когда $f$ принимает целые неотрицательные значения и определена на множестве $\Omega\left(\mathscr{D}_{1}, \ldots, \mathscr{D}_{k}\right)$.

Пусть $H$ - множество различных стохастических векторов. Мы говорим, что стохастический вектор $\mathscr{D}$ порождается множеством $H$, если для некоторой функции $f\left(x_{1}, \ldots, x_{k}\right)$ и некоторых $\mathscr{D}_{1}, \ldots, \mathscr{D}_{k}$ из $H$ выполняется равенство

$$
\mathscr{D}=\mathbf{P}\left\{f\left(\mathscr{D}_{1}, \ldots, \mathscr{D}_{k}\right)\right\} \text {. }
$$

Под замыканием $\langle H\rangle$ множества $H$ мы понимаем множество всех стохастических векторов, порождаемых множеством $H$. Заметим, что $H \subseteq\langle H\rangle$. Кроме того, нетрудно проверить, что для любых множеств $H^{\prime}$ и $H^{\prime \prime}$ стохастических векторов справедливо включение $\left\langle H^{\prime} \cap H^{\prime \prime}\right\rangle \subseteq\left\langle H^{\prime}\right\rangle \cap\left\langle H^{\prime \prime}\right\rangle$. Далее (следствие 1) мы показываем, что $\langle\langle H\rangle\rangle=\langle H\rangle$. Таким образом, рассматриваемая нами операция замыкания множеств стохастических векторов является корректно определенной с точки зрения стандартных свойств операции замыкания. Множество $H$ называется замкнутым, если $\langle H\rangle=H$. Будем также говорить, что множество $A$ стохастических векторов порождается множеством $H$, если $A \subseteq\langle H\rangle$. Поскольку двумерный стохастический вектор однозначно определяется какой-либо одной из его компонент, вместо двумерных стохастических векторов мы можем также рассматривать числа из сегмента $[0,1]$, являющиеся вторыми компонентами этих векторов. Таким образом, для произвольного множества $M$ чисел из сегмента $[0,1]$ мы можем определить замыкание $\langle M\rangle$ этого множества как замыкание множества соответствующих этим числам двумерных стохастических векторов. Данное понятие замыкания числовых множеств совпадает с понятием замыкания множеств, введенным в [4]. Для произвольного множества натуральных чисел $T$ и натурального $k$ мы обозначаем через $T^{>k}$ множество всех чисел из $T$, больших $k$. Для любого натурального числа $n$ обозначим через $\mathscr{I}(n)$ множество всех простых делителей $n$. Кроме того, в работе используются следующие обозначения: $\mathbf{N}$ - множество натуральных чисел; $\mathbf{Z}^{+}-$множество целых неотрицательных чисел; $\operatorname{gcd}\left(x_{1}, \ldots, x_{n}\right)$ - наибольший общий делитель чисел $x_{1}, \ldots, x_{n} ; B_{i}^{k}-i$-й слой единичного куба $\{0,1\}^{k}$, то есть множество всех наборов из $\{0,1\}^{k}$, имеющих ровно $i$ единичных компонент; $|A|$ - число элементов множества $A ;\lceil x\rceil$ - наименьшее целое число, большее или равное $x$.

Для синтеза преобразователей вероятностных распределений большой интерес представляет задача описания замыканий произвольных множеств чисел из интервала $(0,1)$. Принципиальная трудность этой задачи заключается, очевидно, в невозможности непосредственного описания произвольных числовых множеств. Поэтому естественным подходом к ее решению является изучение замыканий не более чем счетных множеств, 
состоящих из чисел, образующих в совокупности некоторое всюду плотное подмножество интервала $(0,1)$. Наиболее подходящим примером таких чисел представляются рациональные числа из интервала $(0,1)$. Множество всех рациональных чисел из интервала $(0,1)$ мы обозначаем через $\mathbf{Q}(0,1)$. Из формул $(2)$ и (1) нетрудно заметить, что множества чисел из $\mathbf{Q}(0,1)$ могут порождать только стохастические векторы с рациональными компонентами. Множество всех таких векторов мы будем обозначать через SQ. Для любого непустого множества П различных простых чисел мы выделяем из SQ подмножество $G[\Pi]$ всех стохастических векторов, все компоненты которых выражаются дробями со знаменателями, являющимися произведениями степеней чисел из П:

$$
\begin{aligned}
& G[\Pi]=\left\{\left(d_{1}, \ldots, d_{h}\right) \mid d_{1}+\ldots+d_{h}=1, d_{i}=m_{i} / n, m_{i} \in \mathbf{Z}^{+}, i=1, \ldots, h,\right. \\
& n \in \mathbf{N}, \mathscr{\{}[n] \subseteq \Pi\} .
\end{aligned}
$$

В $[11,12]$ показано, что множества всех двумерных стохастических векторов из $G[\{2\}]$ и $G[\{3\}]$ порождаются системами чисел $\{1 / 2\}$ и $\{1 / 3,2 / 3\}$ соответственно. В $[7,10]$ данные результаты были обобщены на случай множества всех двумерных стохастических векторов из $G[\Pi]$ для произвольного П. Была также полностью установлена структура решетки, образуемой этими множествами. Аналогичные результаты для случая стохастических векторов произвольной размерности получены в $[8,9]$. Некоторые аспекты приближенного порождения двумерных стохастических векторов рассматривались в $[6,12]$. В [3] дано явное описание замыканий всех конечных множеств чисел из $\mathbf{Q}(0,1)$ в классе всех двумерных стохастических векторов. В [4] явно описаны замыкания всех одноэлементных множеств чисел из $\mathbf{Q}(0,1)$. В настоящей работе мы обобщаем этот результат на случай замыканий всех конечных множеств чисел из $\mathbf{Q}(0,1)$. Полученное нами описание этих замыканий позволяет для любого заданного стохастического вектора и любого заданного конечного множества чисел из $\mathbf{Q}(0,1)$ эффективно определить, порождается ли данный вектор данным множеством.

\section{2. Вспомогательные определения и утверждения}

Прежде всего отметим следующее свойство рассматриваемого порождения стохастических векторов.

Предложение 1. Пусть $\mathscr{D}_{1}^{(1)}, \ldots, \mathscr{D}_{k(1)}^{(1)}, \ldots, \mathscr{D}_{1}^{(n)}, \ldots, \mathscr{D}_{k(n)}^{(n)}-$ cтохастические векторы $u$ $f_{0}\left(x_{1}, \ldots, x_{n}\right), f_{1}\left(x_{1}, \ldots, x_{k(1)}\right), \ldots, f_{n}\left(x_{1}, \ldots, x_{k(n)}\right)$ - функчии такие, что определень векторы $\mathbf{P}\left\{f_{1}\left(\mathscr{D}_{1}^{(1)}, \ldots, \mathscr{D}_{k(1)}^{(1)}\right)\right\}, \ldots, \mathbf{P}\left\{f_{n}\left(\mathscr{D}_{1}^{(n)}, \ldots, \mathscr{D}_{k(n)}^{(n)}\right)\right\} u$

$$
\mathbf{P}\left\{f_{0}\left(\mathbf{P}\left\{f_{1}\left(\mathscr{D}_{1}^{(1)}, \ldots, \mathscr{D}_{k(1)}^{(1)}\right)\right\}, \ldots, \mathbf{P}\left\{f_{n}\left(\mathscr{D}_{1}^{(n)}, \ldots, \mathscr{D}_{k(n)}^{(n)}\right)\right\}\right)\right\} .
$$

Тогда вектор (3) равен $\mathbf{P}\left\{\hat{f}\left(\mathscr{D}_{1}^{(1)}, \ldots, \mathscr{D}_{k(1)}^{(1)}, \ldots, \mathscr{D}_{1}^{(n)}, \ldots, \mathscr{D}_{k(n)}^{(n)}\right)\right\}$, где

$$
\hat{f}\left(x_{1}^{(1)}, \ldots, x_{k(1)}^{(1)}, \ldots, x_{1}^{(n)}, \ldots, x_{k(n)}^{(n)}\right)=f_{0}\left(f_{1}\left(x_{1}^{(1)}, \ldots, x_{k(1)}^{(1)}\right), \ldots, f_{n}\left(x_{1}^{(n)}, \ldots, x_{k(n)}^{(n)}\right)\right) .
$$

Данное предложение является обобщением утверждения 1 из [3] и доказывается аналогичным образом. Поэтому мы опускаем его доказательство.

Следствие 1. Замыкание любого множества чисел из интервала $(0,1)$ является замкнутым множеством. 
Стохастический вектор называется вырожденным, если он содержит компоненту, равную 1. Мы естественным образом можем считать, что все вырожденные стохастические векторы порождаются пустым множеством и поэтому содержатся в любом замкнутом множестве стохастических векторов. Для любого стохастического вектора $\mathscr{D}$ обозначим через $\mathscr{D}^{+}$стохастический вектор, получающийся из $\mathscr{D}$ удалением всех нулевых компонент. Множество $H$ стохастических векторов назовем позитивно замкнутым, если для любого вектора $\mathscr{D}$ из $H$ вектор $\mathscr{D}^{+}$также содержится в $H$. Очевидно, что любой стохастический вектор $\mathscr{D}$ порождается вектором $\mathscr{D}^{+}$, поэтому справедлив следующий факт.

Предложение 2. Если любой невырожденный вектор с ненулевыми компонентами из позитивно замкнутого множества $G$ стохастических векторов содержится в замкнутом множестве $H$ стохастических векторов, то $G \subseteq H$.

В дальнейшем мы будем обозначать через $\Sigma_{i}(\mathscr{D})$, где $\mathscr{D}-$ произвольный стохастический вектор, сумму $\sum_{j=1}^{i} \mathscr{D}[j]$, при этом для удобства величину $\Sigma_{0}(\mathscr{D})$ будем полагать равной 0. Отметим следующие очевидные свойства стохастических векторов и величины (1).

Предложение 3. Два стохастических вектора $\mathscr{D}_{1}, \mathscr{D}_{2}$ одинаковой размерности $h$ равны тогда и только тогда, когда $\Sigma_{i}\left(\mathscr{D}_{1}\right)=\Sigma_{i}\left(\mathscr{D}_{2}\right)$ для $i=1, \ldots, h-1$.

Предложение 4. Пусть $\mathscr{D}_{1}, \ldots, \mathscr{D}_{k}-$ стохастические векторы, $E_{1}, \ldots, E_{s}$ - непересекаюшиеся подмножества множества $\Omega\left(\mathscr{D}_{1}, \ldots, \mathscr{D}_{k}\right)$. Тогда

$$
\mathbf{P}_{\bigcup_{i=1}^{s} E_{i}}\left(\mathscr{D}_{1}, \ldots, \mathscr{D}_{k}\right)=\sum_{i=1}^{s} \mathbf{P}_{E_{i}}\left(\mathscr{D}_{1}, \ldots, \mathscr{D}_{k}\right)
$$

Отметим также, что в стохастическом векторе $\mathscr{D}=\left(d_{1}, \ldots, d_{h}\right)$ компонента $d_{h}$ однозначно определяется компонентами $d_{1}, \ldots, d_{h-1}$. Поэтому справедливо следующее утверждение.

Предложение 5. Для любых стохастических векторов $\mathscr{D}_{0}, \mathscr{D}_{1}, \ldots, \mathscr{D}_{k}$ соотношение $\mathscr{D}_{0}=\mathbf{P}\left\{f\left(\mathscr{D}_{1}, \ldots, \mathscr{D}_{k}\right)\right\}$ выполняется тогда и только тогда, когда $f$ принимает значения $0,1, \ldots, h_{0}-1$, где $h_{0}-$ размерность вектора $\mathscr{D}_{0}$, и равенства (2) справедливы для $j=1, \ldots, h_{0}-1$.

Пусть $f_{\perp}^{(h)}-$ функция, определенная на $\{0,1, \ldots, h-1\} \times\{0,1, \ldots, h-1\} \times\{0,1\}$ и принимающую на наборе $\left(\sigma_{1}, \sigma_{2}, \sigma_{3}\right)$ из этого множества значение $\sigma_{1}$, если $\sigma_{3}=0$, и значение $\sigma_{2}$, если $\sigma_{3}=1$. Нетрудно проверить, что для любых $h$-мерных стохастических векторов $\mathscr{D}_{1}, \mathscr{D}_{2}$ и двумерного стохастического вектора $\mathscr{D}_{3}$ выполняются соотношения

$$
\begin{aligned}
\mathbf{P}\left\{f_{\perp}^{(h)}\left(\mathscr{D}_{1}, \mathscr{D}_{2}, \mathscr{D}_{3}\right)\right\}[i] & =\mathscr{D}_{3}[1] \mathscr{D}_{1}[i]+\mathscr{D}_{3}[2] \mathscr{D}_{2}[i], & & i=1, \ldots, h, \\
\Sigma_{i}\left(\mathbf{P}\left\{f_{\perp}^{(h)}\left(\mathscr{D}_{1}, \mathscr{D}_{2}, \mathscr{D}_{3}\right)\right\}\right) & =\mathscr{D}_{3}[1] \Sigma_{i}\left(\mathscr{D}_{1}\right)+\mathscr{D}_{3}[2] \Sigma_{i}\left(\mathscr{D}_{2}\right), & & i=1, \ldots, h-1 .
\end{aligned}
$$

Следуя стандартной терминологии, натуральные числа $a_{1}, a_{2}, \ldots, a_{k}$ назовем попарно простыми, если каждое из этих чисел взаимно просто с любым другим из них. Множество чисел из $\mathbf{N}^{>1}$ будем называть разделимым, если оно содержит меньше двух чисел, либо все его числа попарно просты. Будем также называть множество натуральных чисел взаимно простым с натуральным числом $n$, если любое число из этого множества взаимно просто с $n$. Пустое множество считается взаимно простым с любым натуральным числом. 
Пусть $A_{1}, \ldots, A_{s}$ - конечные разделимые множества. Наибольшим общим делителем $\operatorname{GCD}\left(A_{1}, \ldots, A_{s}\right)$ этих множеств мы называем множество

$$
\left\{a \mid a=\operatorname{gcd}\left(a_{1}, a_{2}, \ldots, a_{s}\right)>1, a_{i} \in A_{i}, i=1,2, \ldots, s\right\},
$$

состоящее из больших единицы наибольших общих делителей всевозможных выборок из $s$ чисел по одному числу от каждого из множеств $A_{1}, \ldots, A_{s}$. Если хотя бы одно из множеств $A_{1}, \ldots, A_{s}$ является пустым, будем полагать $\operatorname{GCD}\left(A_{1}, \ldots, A_{s}\right)=\varnothing$. Отметим следующие очевидные свойства наибольшего общего делителя разделимых множеств.

Предложение 6. Наибольший общий делитель разделимых множеств является разделимым множеством, взаимно простым с любым из чисел, взаимно простых с хотя бы одним из этих множеств.

Отметим также ассоциативность операции взятия наибольшего общего делителя разделимых множеств: для любых разделимых множеств $A, B, C$ выполняются равенства

$$
\mathrm{GCD}(\mathrm{GCD}(A, B), C)=\mathrm{GCD}(A, \mathrm{GCD}(B, C))=\mathrm{GCD}(A, B, C) .
$$

Если множество натуральных чисел $A$ конечно, мы обозначаем через $\|A\|$ произведение всех чисел множества $A$. Для пустого множества полагаем $\|\varnothing\|=1$. Отметим следующие очевидные факты.

Предложение 7. Величина $\|A\|$ взаимно проста с любым натуральным числом, взаимно простым с множеством А.

Предложение 8. Для любых конечных разделимых множеств $A, B$ справедливо соотношение $\|\mathrm{GCD}(A, B)\|=\operatorname{gcd}(\|A\|,\|B\|)$.

Множество натуральных чисел $B=\left\{b_{1}, \ldots, b_{t}\right\}$ будем называть мультипликативным разбиением конечного множества натуральных чисел $A$, если ему соответствует некоторое разбиение множества $A$ на непересекающиеся (возможно несобственные) подмножества $A_{1}, \ldots, A_{t}$ такие, что $b_{i}=\left\|A_{i}\right\|, i=1, \ldots, t$. Заметим, что для любого множества несобственными подмножествами называются пустое подмножество и подмножество, совпадающее с самим этим множеством.

Из предложения 7 вытекает следующее утверждение.

Предложение 9. Мультипликативное разбиение разделимого множества является разделимым и взаимно простым с любым из натуральных чисел, взаимно простых с этим множеством.

В дальнейшем мы также используем следующие известные теоретико-числовые факты (см., например, [2]).

Теорема 1 (теорема Эйлера). $E с л и \operatorname{gcd}(a, m)=1 u m>1, \operatorname{mo} a^{\varphi(m)} \equiv 1(\bmod m)$.

Рассмотрим сравнение первой степени с одним неизвестным

$$
a x \equiv b \quad(\bmod m)
$$

Лемма 1. Сравнение (6) имеет решения тогда и только тогда, когда $b$ делится на $d=\operatorname{gcd}(a, m)$, и в этом случае все решения данного сравнения образуют класс вычетов по модулю $m / d$. 
Согласно лемме 1 в случае, если $b$ делится на $d$, сравнение (6) имеет решение. Это означает, что существуют целые $x$ и $y$ такие, что $a x+m y=b$. Таким образом, мы получаем следующее утверждение.

Следствие 2. Если $a, b, c \in \mathbf{N} u$ и делится на $\operatorname{gcd}(a, b)$, то уравнение $a x+b y=c$ разрешимо в иелых числах.

Из этого следствия нетрудно получить следующее утверждение.

Следствие 3. Если $a, b, c \in \mathbf{N}$ и с делится на $\operatorname{gcd}(a, b)$, то найдутся натуральные $x, y$ maкие, что $x \leqslant b, y \leqslant a u a x+b y \equiv c(\bmod a b)$.

Рассмотрим теперь систему сравнений с одним неизвестным

$$
\begin{gathered}
x \equiv b_{1} \quad\left(\bmod m_{1}\right), \\
x \equiv b_{2} \quad\left(\bmod m_{2}\right), \\
\ldots \\
x \equiv b_{k} \quad\left(\bmod m_{k}\right),
\end{gathered}
$$

где $m_{1}, m_{2}, \ldots, m_{k}-$ попарно простые числа.

Лемма 2. Система (7) разрешима и все ее решения образуют класс вычетов по модулю $m_{1} m_{2} \ldots m_{k}$.

\section{3. Замыкания конечных множеств рациональных чисел}

Пусть $\Pi$ - произвольное непустое множество различных простых чисел, $T$ - конечное разделимое множество натуральных чисел, взаимно простых с множеством П, и $a, b-$ натуральные числа, взаимно простые с множеством П. Определим следующие подмножества множества $G[\Pi]$ (рассматриваемые в определении множества $G[\Pi ; T]$ подмножества $T_{1} \ldots, T_{h-1}$ множества $T$ могут быть несобственными):

$$
\begin{aligned}
& G[\Pi ; T]=\left\{\left(d_{1}, \ldots, d_{h}\right) \mid d_{i}=\frac{m_{i}}{n}, m_{i} \in \mathbf{Z}^{+}, i=1, \ldots, h,\right. \\
& \sum_{i=1}^{h} d_{i}=1, n \in \mathbf{N}, \mathscr{g}[n] \subseteq \Pi, \exists T_{1}, \ldots, T_{h-1}, T \supseteq T_{1} \supseteq T_{2} \supseteq \ldots \supseteq T_{h-1}, \\
& \left.\sum_{j=1}^{i} m_{j} \equiv 0 \quad\left(\bmod \left\|T_{i}\right\|\right), \sum_{j=1}^{i} m_{j} \equiv n \quad\left(\bmod \left\|T \backslash T_{i}\right\|\right), i=1, \ldots, h-1\right\} \\
& G[\Pi ; a: b]=\left\{\left(d_{1}, \ldots, d_{h}\right) \mid d_{i}=\frac{m_{i}}{n}, m_{i} \in \mathbf{Z}^{+}, i=1, \ldots, h, \sum_{i=1}^{h} d_{i}=1, n \in \mathbf{N},\right. \\
& \left.\Im[n] \subseteq \Pi, \sum_{j=1}^{i} m_{j} \equiv 0 \quad(\bmod a), \sum_{j=1}^{i} m_{j} \equiv n \quad(\bmod b), i=1, \ldots, h-1\right\} .
\end{aligned}
$$

В случае $T=\varnothing$ мы полагаем $G[\Pi ; \varnothing]=G[\Pi]$. Принадлежность стохастического вектора множеству $G[\Pi ; T]$ формально зависит от выбора общего знаменателя $n$ его компонент. 
Однако в [4] показано, что эта зависимость является фиктивной, то есть стохастический вектор принадлежит множеству $G[\Pi ; T]$ независимо от выбора знаменателя $n$. Аналогичным образом можно показать, что данное свойство также справедливо для множества $G[\Pi ; a: b]$. Заметим, что поскольку любое число $t$ из $T$ взаимно просто с множеством $\Pi$ и, следовательно, взаимно просто с $n$, соотношения

$$
\sum_{j=1}^{i} m_{j} \equiv 0 \quad(\bmod t), \quad \sum_{j=1}^{i} m_{j} \equiv n \quad(\bmod t)
$$

не могут быть выполнены одновременно. Поэтому для любого вектора $\left(d_{1}, \ldots, d_{h}\right)$ из $G[\Pi ; T]$ соответствующие подмножества $T_{1} \ldots, T_{h-1}$ однозначно определяются этим вектором. Кроме того, отметим, что любое множество $G[\Pi ; T]$ является позитивно замкнутым и содержит все вырожденные стохастические векторы. Можно доказать, что любое множество $G[\Pi ; T]$ является также замкнутым (см. [5]) и, в частности, инвариантным относительно перестановок компонент входящих в него стохастических векторов.

Основной целью данной работы является описание замыканий конечных множеств чисел из $\mathbf{Q}(0,1)$. Поскольку любое рациональное число однозначно выражается несократимой дробью, без ограничения общности мы можем рассматривать только замыкания конечных множеств несократимых дробей из интервала $(0 ; 1)$.

Пусть $M=\left\{m_{1} / n_{1}, \ldots, m_{s} / n_{s}\right\}$ - произвольное конечное множество несократимых дробей из $\mathbf{Q}(0,1)$. Положим

$$
\Pi(M)=\bigcup_{i=1}^{s} \mathscr{T}\left(n_{i}\right)
$$

и

$$
T(M)= \begin{cases}\operatorname{GCD}\left(\left\{m_{1}, n_{1}-m_{1}\right\}^{>1}, \ldots,\left\{m_{s}, n_{s}-m_{s}\right\}^{>1}\right), & \text { если } s \geqslant 2, \\ \left\{m_{1}, n_{1}-m_{1}\right\}^{>1}, & \text { если } s=1 .\end{cases}
$$

Так как дроби из $M$ являются несократимыми, для любого $i=1, \ldots, s$ множество $\left\{m_{i}, n_{i}-m_{i}\right\}^{>1}$ является разделимым и взаимно простым с $n_{i}$. Следовательно, согласно предложению 6 множество $T(M)$ обладает следующими свойствами.

Предложение 10. Множество $T(M)$ является разделимым и взаимно простым с каждым из чисел $n_{1}, \ldots, n_{s}$.

Поэтому мы можем рассмотреть множество $G[\Pi(M) ; T(M)]$. В [4] получен следующий результат.

Теорема 2. Пусть $m / n-$ произвольная несократимая дробь из $\mathbf{Q}(0,1)$. Тогда

$$
\langle\{m / n\}\rangle=G\left[\Phi(n) ;\{m, n-m\}^{>1}\right] .
$$

Главным результатом данной работы является следующее обобщение теоремы 2 на случай произвольных конечных подмножеств множества $\mathbf{Q}(0,1)$.

Теорема 3. Пусть $M=\left\{m_{1} / n_{1}, \ldots, m_{s} / n_{s}\right\}$-конечное множество несократимых дробей из $\mathbf{Q}(0 ; 1)$. Тогда $\langle M\rangle=G[\Pi(M) ; T(M)]$.

Дальнейшая часть статьи посвящена доказательству теоремы 3. 


\section{4. Вспомогательные результаты}

Пусть $p, q$ - два, возможно одинаковых, простых числа, $a, b$ - натуральные числа такие, что $\operatorname{gcd}(a, p)=\operatorname{gcd}(b, q)=1$. Тогда мы можем рассмотреть множества стохастических векторов $G[\{p\} ; a: 1]$ и $G[\{q\} ; b: 1]$. Положим $l=\operatorname{gcd}(a, b)$. Поскольку $(p, l)=1$, мы можем также рассмотреть множество стохастических векторов $G[\{p\} ; l: 1]$.

Лемма 3. Пусть $H$ - замкнутое множество стохастических векторов, содержащее множества $G[\{p\} ; a: 1] u G[\{q\} ; b: 1]$. Тогда $G[\{p\} ; l: 1] \subseteq H$.

Доказательство. Рассмотрим произвольный стохастический вектор $\mathscr{D}=\left(d_{1}, \ldots, d_{h}\right)$ из $G[\{p\} ; l: 1]$. Покажем, что $\mathscr{D} \in H$. Без ограничения общности мы можем рассматривать числа $d_{1}, \ldots, d_{h}$ как некоторые дроби с общим знаменателем $n$, являющимся степенью числа $p$. Обозначим через $\hat{p}$ число $p^{\varphi(a)}$ и через $\hat{q}$ число $q^{\varphi(b)}$. Отметим, что согласно теореме Эйлера $\hat{p} \equiv 1(\bmod a)$ и $\hat{q} \equiv 1(\bmod b)$. Выберем в качестве числа $n_{1}$ достаточно большую степень числа $\hat{p}$, не меньшую, чем $n$, и удовлетворяющую условиям

$$
\begin{aligned}
& n_{1}>a b(h-1), \\
& n_{1}>\frac{2}{d_{h}} .
\end{aligned}
$$

Возьмем в качестве числа $n_{2}$ достаточно большую степень числа $\hat{q}$ такую, что

$$
\begin{aligned}
& n_{2}>a b h, \\
& n_{2}>\frac{2}{d_{h}} .
\end{aligned}
$$

Заметим, что $n_{1} \equiv 1(\bmod a)$ и $n_{2} \equiv 1(\bmod b)$. Представим числа $d_{1}, \ldots, d_{h}$ в виде дробей $m_{1} /\left(n_{1} n_{2}\right), \ldots, m_{h} /\left(n_{1} n_{2}\right)$ соответственно. Так как $\mathscr{D} \in G[\{p\} ; l: 1]$, выполняются соотношения

$$
m_{i} \equiv 0 \quad(\bmod l), \quad i=1, \ldots, h-1 .
$$

Поэтому согласно следствию 3 для каждого $i=1, \ldots, h-1$ существуют натуральные $\alpha_{i}, \beta_{i}$ такие, что $\alpha_{i} \leqslant b, \beta_{i} \leqslant a$ и справедливо соотношение

$$
\alpha_{i} a+\beta_{i} b \equiv m_{i} \quad(\bmod a b)
$$

так что число $\left(m_{i}-\alpha_{i} a-\beta_{i} b\right) /(a b)$ является целым. Обозначим это число через $m_{i}^{\prime}$. Поскольку $\alpha_{i} \leqslant b$ и $\beta_{i} \leqslant a$, то

$$
\alpha_{i} a+\beta_{i} b \leqslant 2 a b
$$

С другой стороны, поскольку $d_{i} \geqslant 1 / n \geqslant 1 / n_{1}$, то $m_{i}=d_{i} n_{1} n_{2} \geqslant n_{2}$. Поэтому из неравенства (10) следует, что $m_{i}>a b h \geqslant 2 a b$. Сопоставляя это соотношение с неравенством (13), получим, что $m_{i}^{\prime}>0$.

Обозначим через $k_{1}$ целое число $\left(n_{1}-1\right) / a$ и через $k_{2}$ целое число $\left(n_{2}-1\right) / b$. Из неравенств (8) и (10) вытекает, что

$$
k_{1} \geqslant b(h-1), \quad k_{2} \geqslant a h .
$$


Рассмотрим стохастические векторы

$$
\mathscr{D}_{1}=\left(\frac{a}{n_{1}}, \ldots, \frac{a}{n_{1}}, \frac{1}{n_{1}}\right), \quad \mathscr{D}_{2}=\left(\frac{b}{n_{2}}, \ldots, \frac{b}{n_{2}}, \frac{1}{n_{2}}\right),
$$

размерности $k_{1}+1$ и $k_{2}+1$ соответственно. Заметим, что $\mathscr{D}_{1} \in G[\{p\} ; a: 1]$ и $\mathscr{D}_{2} \in G[\{q\} ; b: 1]$, поэтому $\mathscr{D}_{1}, \mathscr{D}_{2} \in H$.

Выделим в множестве $\Omega\left(\mathscr{D}_{1}, \mathscr{D}_{2}\right)$ непересекающиеся подмножества

$$
\begin{aligned}
& \Omega_{0}=\left\{\left(\sigma_{1}, \sigma_{2}\right) \mid\left(\sigma_{1}, \sigma_{2}\right) \in \Omega\left(\mathscr{D}_{1}, \mathscr{D}_{2}\right), \sigma_{1}<k_{1}, \sigma_{2}<k_{2}\right\}, \\
& \Omega_{1}=\left\{\left(\sigma_{1}, \sigma_{2}\right) \mid\left(\sigma_{1}, \sigma_{2}\right) \in \Omega\left(\mathscr{D}_{1}, \mathscr{D}_{2}\right), \sigma_{1}<k_{1}, \sigma_{2}=k_{2}\right\}, \\
& \Omega_{2}=\left\{\left(\sigma_{1}, \sigma_{2}\right) \mid\left(\sigma_{1}, \sigma_{2}\right) \in \Omega\left(\mathscr{D}_{1}, \mathscr{D}_{2}\right), \sigma_{1}=k_{1}, \sigma_{2}<k_{2}\right\} .
\end{aligned}
$$

Заметим, что для любых подмножеств $U_{0}, U_{1}, U_{2}$ множеств $\Omega_{0}, \Omega_{1}, \Omega_{2}$, соответственно, справедливы равенства

$$
\mathbf{P}_{U_{0}}\left(\mathscr{D}_{1}, \mathscr{D}_{2}\right)=\frac{a b\left|U_{0}\right|}{n_{1} n_{2}}, \quad \mathbf{P}_{U_{1}}\left(\mathscr{D}_{1}, \mathscr{D}_{2}\right)=\frac{a\left|U_{1}\right|}{n_{1} n_{2}}, \quad \mathbf{P}_{U_{2}}\left(\mathscr{D}_{1}, \mathscr{D}_{2}\right)=\frac{b\left|U_{2}\right|}{n_{1} n_{2}},
$$

поэтому для любого подмножества $U$ множества $\Omega_{0} \cup \Omega_{1} \cup \Omega_{2}$

$$
\mathbf{P}_{U}\left(\mathscr{D}_{1}, \mathscr{D}_{2}\right)=\frac{a b\left|U \cap \Omega_{0}\right|+a\left|U \cap \Omega_{1}\right|+b\left|U \cap \Omega_{2}\right|}{n_{1} n_{2}}
$$

Построим функцию $f: \Omega\left(\mathscr{D}_{1}, \mathscr{D}_{2}\right) \rightarrow\{0,1, \ldots, h-1\}$ такую, что

$$
\mathscr{D}=\mathbf{P}\left\{f\left(\mathscr{D}_{1}, \mathscr{D}_{2}\right)\right\} .
$$

Последовательно определим непересекающиеся множества $\mathcal{N}_{0}(f), \ldots, \mathcal{N}_{h-2}(f)$ такие, что

$$
\mathbf{P}_{\mathcal{N}_{i}(f)}\left(\mathscr{D}_{1}, \mathscr{D}_{2}\right)=\frac{m_{i+1}}{n_{1} n_{2}}, \quad i=0,1, \ldots, h-2 .
$$

В процессе построения этих множеств будем дополнительно требовать, чтобы для любого $i=0,1, \ldots, h-2$ выполнялись неравенства

$$
\left|\Omega_{1} \backslash \bigcup_{j<i} \mathcal{N}_{j}(f)\right| \geqslant((h-1)-i) b, \quad\left|\Omega_{2} \backslash \bigcup_{j<i} \mathcal{N}_{j}(f)\right| \geqslant((h-1)-i) a .
$$

Отметим, что при $i=0$

$$
\left|\Omega_{s} \backslash \bigcup_{j<i} \mathcal{N}_{j}(f)\right|=\left|\Omega_{s} \backslash \varnothing\right|=\left|\Omega_{s}\right|=k_{s}
$$

для $s=1,2$, поэтому в этом случае соотношения (18) следуют из неравенств (14).

Пусть для некоторого $i \in\{0,1, \ldots, h-2\}$ нами уже построены все искомые множества $\mathcal{N}_{j}(f)$, где $j<i$. Обозначим через $\Theta$ объединение этих множеств. Согласно утверждению 4

$$
\mathbf{P}_{\Theta}\left(\mathscr{D}_{1}, \mathscr{D}_{2}\right)=\sum_{j<i} \mathbf{P}_{\mathcal{N}_{j}(f)}\left(\mathscr{D}_{1}, \mathscr{D}_{2}\right)=\sum_{j \leqslant i} \frac{m_{j}}{n_{1} n_{2}}
$$


Положим $V=\Theta \cap \Omega_{0}$. Применяя формулу (15) для $V$, получим, что

$$
\mathbf{P}_{V}\left(\mathscr{D}_{1}, \mathscr{D}_{2}\right)=\frac{a b|V|}{n_{1} n_{2}}
$$

Так как $V \subseteq \Theta$, то $\mathbf{P}_{V}\left(\mathscr{D}_{1}, \mathscr{D}_{2}\right) \leqslant \mathbf{P}_{\Theta}\left(\mathscr{D}_{1}, \mathscr{D}_{2}\right)$, поэтому из равенств (19) и (20) следует, что

$$
|V| \leqslant \frac{1}{a b} \sum_{j \leqslant i} m_{j}
$$

Заметим также, что

$$
\left|\Omega_{0}\right|=k_{1} k_{2}=\frac{n_{1}-1}{a} \frac{n_{2}-1}{b}>\frac{n_{1} n_{2}}{a b}\left(1-\frac{1}{n_{1}}-\frac{1}{n_{2}}\right) .
$$

Поэтому из неравенств (9) и (11) следует, что

$$
\left|\Omega_{0}\right|>\frac{n_{1} n_{2}}{a b}\left(1-d_{h}\right)=\frac{1}{a b} \sum_{j=1}^{h-1} m_{j} .
$$

Из этого неравенства в совокупности с неравенством (21) получаем, что

$$
\left|\Omega_{0}\right|-|V|>\frac{1}{a b}\left(\sum_{j=1}^{h-1} m_{j}-\sum_{j \leqslant i} m_{j}\right) \geqslant \frac{1}{a b} m_{i+1} .
$$

Тогда, поскольку $m_{i+1}^{\prime}<m_{i+1} / a b$, справедливо неравенство

$$
m_{i+1}^{\prime}<\left|\Omega_{0}\right|-|V|=\left|\Omega_{0} \backslash \Theta\right| .
$$

Кроме того, из справедливости неравенств (18) для $i$ следует, что

$$
\left|\Omega_{1} \backslash \Theta\right| \geqslant b \geqslant \alpha_{i+1}, \quad\left|\Omega_{2} \backslash \Theta\right| \geqslant a \geqslant \beta_{i+1} .
$$

Таким образом, мы можем взять в качестве $\mathcal{N}_{i}(f)$ множество, состоящее из произвольных $m_{i+1}^{\prime}$ наборов из множества $\Omega_{0} \backslash \Theta$, произвольных $\alpha_{i+1}$ наборов из множества $\Omega_{1} \backslash \Theta$ и произвольных $\beta_{i+1}$ наборов из множества $\Omega_{2} \backslash \Theta$. Согласно формуле (15) для $\mathcal{N}_{i}(f)$ справедливо равенство

$$
\mathbf{P}_{\mathcal{N}_{i}(f)}\left(\mathscr{D}_{1}, \mathscr{D}_{2}\right)=\frac{a b m_{i+1}^{\prime}+a \alpha_{i+1}+b \beta_{i+1}}{n_{1} n_{2}}=\frac{m_{i+1}}{n_{1} n_{2}} .
$$

Отметим также, что из справедливости неравенств (18) для $i$ и неравенств $\alpha_{i+1} \leqslant b$, $\beta_{i+1} \leqslant a$ вытекает справедливость неравенств (18) для $i+1$.

Завершив построение множеств $\mathcal{N}_{0}(f), \ldots, \mathcal{N}_{h-2}(f)$, положим

$$
\mathcal{N}_{h-1}(f)=\Omega\left(\mathscr{D}_{1}, \mathscr{D}_{2}\right) \backslash \bigcup_{j=0}^{h-2} \mathcal{N}_{j}(f)
$$

Тогда в силу предложения 5 из равенств (17) следует, что функция $f$ удовлетворяет соотношению (16). Следовательно, поскольку $\mathscr{D}_{1}, \mathscr{D}_{2} \in H$ и $H$ замкнуто, справедливо включение $\mathscr{D} \in H$. Лемма доказана. 
Пусть $p$ - простое число, $A$ - конечное разделимое множество чисел, взаимно простых с $p, b$ - натуральное число, взаимно простое с $p$. Тогда можно рассмотреть множества стохастических векторов $G[\{p\} ; A]$ и $G[\{p\} ; b: 1]$. Согласно предложению 6 множество $\operatorname{GCD}\left(A,\{b\}^{>1}\right)$ является разделимыми и взаимно простым с $p$, поэтому мы можем также рассмотреть множество стохастических векторов $G\left[\{p\} ; \operatorname{GCD}\left(A,\{b\}^{>1}\right)\right]$.

Лемма 4. Пусть $\mathrm{H}$ - замкнутое множество стохастических векторов, содержащее множества $G[\{p\} ; A] u G[\{p\} ; b: 1]$. Тогда $G\left[\{p\} ; \operatorname{GCD}\left(A,\{b\}^{>1}\right)\right] \subseteq H$.

Доказательство. Если $b=1$, то $G[\{p\} ; b: 1]=G[\{p\}]=G\left[\{p\} ; \operatorname{GCD}\left(A,\{b\}^{>1}\right)\right]$, и следовательно, утверждение леммы в этом случае очевидно. Поэтому мы можем предполагать, что $b>1$. Положим $\hat{l}=\|A\|$ и $l=\operatorname{gcd}(\|A\|, b)$. Обозначим через $\hat{p}$ число $p^{\varphi(\hat{l})}$. Отметим, что согласно теореме Эйлера $\hat{p} \equiv 1(\bmod \hat{l})$. Рассмотрим произвольный стохастический вектор $\mathscr{D}=\left(d_{1}, \ldots, d_{h}\right)$ из $G[\{p\} ; \operatorname{GCD}(A,\{b\})]$. Покажем, что $\mathscr{D} \in H$. Это соотношение для случая $h=2$ следует из леммы 9 в [3], поэтому будем предполагать, что $h \geqslant 3$. Выберем в качестве числа $n_{2}$ достаточно большую степень числа $\hat{p}$ такую, что

$$
n_{2}>\hat{l}(h-1) \text {. }
$$

Без ограничения общности мы можем рассматривать числа $d_{1}, \ldots, d_{h}$ как некоторые дроби с общим знаменателем $n$, являющимся степенью числа $p$. Выберем в качестве числа $n_{1}$ достаточно большую степень числа $\hat{p}$, не меньшую, чем $n$, и удовлетворяющую условиям

$$
\begin{aligned}
& n_{1}>n_{2} l(h-1), \\
& n_{1}>\frac{1}{d_{h}} .
\end{aligned}
$$

Заметим, что $n_{1} \equiv n_{2} \equiv 1(\bmod \hat{l})$. Представим числа $d_{1}, \ldots, d_{h}$ в виде дробей $m_{1} /\left(n_{1} n_{2}\right), \ldots, m_{h} /\left(n_{1} n_{2}\right)$ соответственно. Положим

$$
\mu_{0}=0, \quad \mu_{i}=\sum_{j=1}^{i} m_{j}, \quad i=1, \ldots, h-1 .
$$

Так как $\mathscr{D} \in G[\{p\} ; \operatorname{GCD}(A,\{b\})]$, существуют подмножества $T_{1}, \ldots, T_{h-1}$ множества $\operatorname{GCD}(A,\{b\})$ такие, что справедливы соотношения

$$
\begin{array}{rlrl}
\mu_{i} & \equiv 0 \quad\left(\bmod \left\|T_{i}\right\|\right), & & i=1, \ldots, \dot{h}-1, \\
\mu_{i} \equiv n_{1} n_{2} \quad\left(\bmod \left\|\operatorname{GCD}(A,\{b\}) \backslash T_{i}\right\|\right), & & i=1, \ldots, h-1, \\
T_{1} \supseteq T_{2} \supseteq \ldots \supseteq T_{h-1} . & &
\end{array}
$$

Для $i=1, \ldots, h-1$ положим $l_{i}^{\prime}=\left\|T_{i}\right\|$ и $l_{i}^{\prime \prime}=\left\|\mathrm{GCD}(A,\{b\}) \backslash T_{i}\right\|$. Согласно предложению 9 наибольший общий делитель $\operatorname{gcd}\left(l_{i}^{\prime}, l_{i}^{\prime \prime}\right)=1$. Кроме того, $l=\|\operatorname{GCD}(A,\{b\})\|$ в силу предложения 8, поэтому $l=l_{i}^{\prime} l_{i}^{\prime \prime}$.

Для каждого $i=1, \ldots, h-1$ обозначим $\hat{T}_{i}$ подмножество $\left\{a \mid a \in A, \operatorname{gcd}(a, b) \in T_{i}\right\}$ множества $A$. Положим $\hat{l}_{i}^{\prime}=\left\|\hat{T}_{i}\right\|$ и $\hat{l}_{i}^{\prime \prime}=\left\|A \backslash \hat{T}_{i}\right\|$. Заметим, что $l_{i}^{\prime}$ является делителем $\hat{l}_{i}^{\prime}$ и $l_{i}^{\prime \prime}$ является делителем $\hat{l}_{i}^{\prime \prime}$. Отметим также, что $\hat{l}=\hat{l}_{i}^{\prime} \hat{l}_{i}^{\prime \prime}$. Рассмотрим систему сравнений

$$
\begin{array}{ll}
x \equiv 0 & \left(\bmod \hat{l}_{i}^{\prime}\right), \\
x \equiv 1 & \left(\bmod \hat{l}_{i}^{\prime \prime}\right) .
\end{array}
$$


В силу предложения $9 \operatorname{gcd}\left(\hat{l}_{i}^{\prime}, \hat{l}_{i}^{\prime \prime}\right)=1$, поэтому согласно лемме 2 эта система имеет решение, являющееся классом вычетов по модулю $\hat{l}_{i}^{\prime} \hat{l}_{i}^{\prime \prime}=\hat{l}$. Обозначим через $\nu_{i}$ наименьший положительный вычет из данного класса и положим $\mu_{i}^{\prime}=v_{i}+(i-1) \hat{l}$. Так как

$$
0<v_{i} \leqslant \hat{l}, \quad i=1, \ldots, h-1,
$$

справедливо неравенство $0<\mu_{1}^{\prime}$ и $\mu_{i}^{\prime} \leqslant i \hat{l}<\mu_{i+1}^{\prime}$ для каждого $i=1, \ldots, h-2$. Кроме того, учитывая также неравенство (23), получим, что $\mu_{h-1}^{\prime} \leqslant(h-1) \hat{l}<n_{2}$. Таким образом, справедливы неравенства

$$
0<\frac{\mu_{1}^{\prime}}{n_{2}}<\frac{\mu_{2}^{\prime}}{n_{2}}<\ldots<\frac{\mu_{h-1}^{\prime}}{n_{2}}<1 .
$$

Поэтому мы можем рассмотреть стохастический вектор $\mathscr{D}_{1}=\left(m_{1}^{\prime} / n_{2}, \ldots, m_{h}^{\prime} / n_{2}\right)$ такой, что

$$
\sum_{j=1}^{i} m_{j}^{\prime}=\mu_{i}^{\prime}, \quad i=1, \ldots, h-1 .
$$

Для каждого $i=1, \ldots, h-1$ справедливо сравнение $\mu_{i}^{\prime} \equiv v_{i}(\bmod \hat{l})$, поэтому $\mu_{i}^{\prime}$ удовлетворяет системе (29), то есть

$$
\begin{array}{ll}
\mu_{i}^{\prime} \equiv 0 & \left(\bmod \hat{l}_{i}^{\prime}\right) \\
\mu_{i}^{\prime} \equiv 1 & \left(\bmod \hat{l}_{i}^{\prime \prime}\right) .
\end{array}
$$

Из последнего соотношения получаем, что

$$
\mu_{i}^{\prime} \equiv n_{2} \quad\left(\bmod \hat{l}_{i}^{\prime \prime}\right)
$$

Из соотношений (28) очевидным образом вытекают соотношения $\hat{T}_{1} \supseteq \hat{T}_{2} \supseteq \ldots \supseteq \hat{T}_{h-1}$. Из этих соотношений и сравнений (31) и (33) следует, что $\mathscr{D}_{1} \in G[\{p\} ; A]$, то есть $\mathscr{D}_{1} \in H$.

Из соотношения (31) вытекает, что $\mu_{i}^{\prime} \equiv 0\left(\bmod l_{i}^{\prime}\right)$. Сопоставив это сравнение со сравнениями (26), получим, что

$$
\mu_{i}^{\prime} \equiv \mu_{i} \quad\left(\bmod l_{i}^{\prime}\right)
$$

Из соотношений (27) следует, что $\mu_{i} \equiv 1\left(\bmod l_{i}^{\prime \prime}\right)$. Сопоставив это сравнение с вытекающим из соотношения (32) сравнением $\mu_{i}^{\prime} \equiv 1\left(\bmod l_{i}^{\prime \prime}\right)$, получим, что

$$
\mu_{i}^{\prime} \equiv \mu_{i} \quad\left(\bmod l_{i}^{\prime \prime}\right)
$$

Из сравнений (34) и (35), учитывая, что $\operatorname{gcd}\left(l_{i}^{\prime}, l_{i}^{\prime \prime}\right)=1$, получаем соотношения

$$
\mu_{i}^{\prime} \equiv \mu_{i} \quad\left(\bmod l_{i}^{\prime} l_{i}^{\prime \prime}=l\right), \quad i=1, \ldots, h-1 .
$$

Эти соотношения эквивалентны сравнениям

$$
m_{i}^{\prime} \equiv m_{i} \quad(\bmod l), \quad i=1, \ldots, h-1 .
$$

Таким образом, для любого $i=1, \ldots, h-1$ число $\left(m_{i}-m_{i}^{\prime}\right) / l$ является целым. Обозначим это число через $m_{i}^{\prime \prime}$. Полагая $\mu_{0}^{\prime}=0$ и используя неравенства (30), получим, что

$$
m_{i}^{\prime}=\mu_{i}^{\prime}-\mu_{0}^{\prime}<\hat{l}+v_{i} \leqslant 2 \hat{l}, \quad i=1, \ldots, h-1 .
$$


Поэтому, применяя неравенство (23) и учитывая, что $h \geqslant 3$, находим, что $m_{i}^{\prime}<n_{2}$. С другой стороны, поскольку $d_{i} \geqslant 1 / n \geqslant 1 / n_{1}$, то $m_{i}=d_{i} n_{1} n_{2} \geqslant n_{2}$. Таким образом, $m_{i}>m_{i}^{\prime}$, так что $m_{i}^{\prime \prime}>0$ для $i=1, \ldots, h-1$. Поэтому для каждого $i=1, \ldots, h-1$ можно представить $m_{i}^{\prime \prime}$ в виде $m_{i}^{\prime \prime}=\alpha_{i}^{\prime}\left(n_{2}-1\right)+\alpha_{i}^{\prime \prime}$, где $\alpha_{i}^{\prime}, \alpha_{i}^{\prime \prime} \in \mathbf{Z}^{+}$и $\alpha_{i}^{\prime \prime}<n_{2}$.

Обозначим целое число $\left(n_{1}-1\right) / l$ через $k$. Рассмотрим двумерный и $(k+1)$-мерный стохастические векторы

$$
\mathscr{D}_{2}=\left(\frac{n_{2}-1}{n_{2}}, \frac{1}{n_{2}}\right), \quad \mathscr{D}_{3}=\left(\frac{l}{n_{1}}, \ldots, \frac{l}{n_{1}}, \frac{1}{n_{1}}\right) .
$$

Заметим, что оба эти вектора принадлежат множеству $G[\{p\} ; l: 1]$. Отметим, что $G[\{p\} ; b: 1] \subseteq H$, и поскольку $G[\{p\} ;\|A\|: 1] \subseteq G[\{p\} ; A]$, то $G[\{p\} ;\|A\|: 1] \subseteq M$. Поэтому согласно лемме 3 справедливо включение $G[\{p\} ; l: 1] \subseteq H$. Таким образом, $\mathscr{D}_{2}, \mathscr{D}_{3} \in H$.

Построим функщию $f: \Omega\left(\mathscr{D}_{1}, \mathscr{D}_{2}, \mathscr{D}_{3}\right) \rightarrow\{0,1, \ldots, h-1\}$ такую, что

$$
\mathscr{D}=\mathbf{P}\left\{f\left(\mathscr{D}_{1}, \mathscr{D}_{2}, \mathscr{D}_{3}\right)\right\} \text {. }
$$

Последовательно определим непересекающиеся множества $\mathcal{N}_{0}(f), \ldots, \mathcal{N}_{h-2}(f)$ такие, что

$$
\mathbf{P}_{\mathcal{N}_{i}(f)}\left(\mathscr{D}_{1}, \mathscr{D}_{2}, \mathscr{D}_{3}\right)=\frac{m_{i+1}}{n_{1} n_{2}}, \quad i=0,1, \ldots, h-2 .
$$

Изначально положим $\gamma_{0}^{\prime}=\gamma_{0}^{\prime \prime}=0$. Отметим, что тогда $\gamma_{0}^{\prime}<k$ и для $i=0$ справедливо неравенство

$$
\gamma_{i}^{\prime \prime} \leqslant i n_{2}
$$

Пусть для некоторого $i \in\{0,1, \ldots, h-2\}$ нами уже построены все искомые множества $\mathcal{N}_{j}(f)$, где $j<i$. Обозначим через $\Theta$ объединение этих множеств. Согласно предложению 4

$$
\mathbf{P}_{\Theta}\left(\mathscr{D}_{1}, \mathscr{D}_{2}, \mathscr{D}_{3}\right)=\sum_{j<i} \mathbf{P}_{\mathcal{N}_{j}(f)}\left(\mathscr{D}_{1}, \mathscr{D}_{2}, \mathscr{D}_{3}\right)=\sum_{j \leqslant i} \frac{m_{j}}{n_{1} n_{2}}=\frac{\mu_{i}}{n_{1} n_{2}} .
$$

Построим множество $\mathcal{N}_{i}(f)$, рассмотрев два возможных случая.

1. Пусть $\gamma_{i}^{\prime}<k$ и $\gamma_{i}^{\prime \prime}$ удовлетворяет неравенству (38). Положим $\gamma_{i+1}^{\prime}=\gamma_{i}^{\prime}+\alpha_{i+1}^{\prime}$. Выделим два возможных подслучая.

a) Пусть $\gamma_{i+1}^{\prime}<k$. Тогда положим $\gamma_{i+1}^{\prime \prime}=\gamma_{i}^{\prime \prime}+\alpha_{i+1}^{\prime \prime}$. Из неравенства (38) для $\gamma_{i}^{\prime \prime}$ и неравенства $\alpha_{i+1}^{\prime \prime}<n_{2}$ следует справедливость неравенства (38) для $\gamma_{i+1}^{\prime \prime}$. Поэтому $\gamma_{i+1}^{\prime \prime} \leqslant(h-1) n_{2}$. Следовательно, из неравенства (24) вытекает, что $\gamma_{i+1}^{\prime \prime}<n_{1} / l$. Таким образом, $\gamma_{i+1}^{\prime \prime} \leqslant k$. Возьмем в качестве $\mathcal{N}_{i}(f)$ множество всех наборов $\left(\sigma_{1} ; \sigma_{2} ; \sigma_{3}\right)$ таких, что либо $\sigma_{2}=0$ и $\gamma_{i}^{\prime} \leqslant \sigma_{3}<\gamma_{i+1}^{\prime}$, либо $\sigma_{2}=1$ и $\gamma_{i}^{\prime \prime} \leqslant \sigma_{3}<\gamma_{i+1}^{\prime \prime}$, либо $\sigma_{3}=k$ и $\sigma_{1}=i$. Заметим, что тогда

$$
\begin{aligned}
\mathbf{P}_{\mathcal{N}_{i}(f)}\left(\mathscr{D}_{1}, \mathscr{D}_{2}, \mathscr{D}_{3}\right) & =\frac{n_{2}-1}{n_{2}}\left(\Sigma_{\gamma_{i+1}^{\prime}}\left(\mathscr{D}_{3}\right)-\Sigma_{\gamma_{i}^{\prime}}\left(\mathscr{D}_{3}\right)\right)+\frac{1}{n_{2}}\left(\Sigma_{\gamma_{i+1}^{\prime \prime}}\left(\mathscr{D}_{3}\right)-\Sigma_{\gamma_{i}^{\prime \prime}}\left(\mathscr{D}_{3}\right)\right)+\frac{m_{i+1}^{\prime}}{n_{1} n_{2}} \\
& =\frac{n_{2}-1}{n_{2}}\left(\frac{l \gamma_{i+1}^{\prime}}{n_{1}}-\frac{l \gamma_{i}^{\prime}}{n_{1}}\right)+\frac{1}{n_{2}}\left(\frac{l \gamma_{i+1}^{\prime \prime}}{n_{1}}-\frac{l \gamma_{i}^{\prime \prime}}{n_{1}}\right)+\frac{m_{i+1}^{\prime}}{n_{1} n_{2}} \\
& =\frac{\left(n_{2}-1\right) l \alpha_{i+1}^{\prime}+l \alpha_{i+1}^{\prime \prime}+m_{i+1}^{\prime}}{n_{1} n_{2}}=\frac{l m_{i+1}^{\prime \prime}+m_{i+1}^{\prime}}{n_{1} n_{2}}=\frac{m_{i+1}}{n_{1} n_{2}} .
\end{aligned}
$$


б) Пусть $\gamma_{i+1}^{\prime} \geqslant k$. Тогда для каждого $j>i+1$ голожим $\gamma_{j}^{\prime}=k$. Обозначив через $\hat{\alpha}_{i+1}^{\prime \prime}$ число $m_{i+1}^{\prime \prime}-\left(k-\gamma_{i}^{\prime}\right)\left(n_{2}-1\right)$, положим также $\gamma_{i+1}^{\prime \prime}=\gamma_{i}^{\prime \prime}+\hat{\alpha}_{i+1}^{\prime \prime}$. Обозначим через $V$ множество наборов $\left(\sigma_{1}, \sigma_{2}, \sigma_{3}\right)$ из $\Omega$ таких, что либо $\sigma_{2}=0$ и $\sigma_{3}<\gamma_{i}^{\prime}$, либо $\sigma_{2}=1$ и $\sigma_{3}<\gamma_{i}^{\prime \prime}$. Отметим, что

$$
\begin{aligned}
\mathbf{P}_{V}\left(\mathscr{D}_{1}, \mathscr{D}_{2}, \mathscr{D}_{3}\right) & =\frac{n_{2}-1}{n_{2}} \Sigma_{\gamma_{i}^{\prime}}\left(\mathscr{D}_{3}\right)+\frac{1}{n_{2}} \Sigma_{\gamma_{i}^{\prime \prime}}\left(\mathscr{D}_{3}\right) \\
& =\frac{n_{2}-1}{n_{2}} \frac{l \gamma_{i}^{\prime}}{n_{1}}+\frac{1}{n_{2}} \frac{l \gamma_{i}^{\prime \prime}}{n_{1}}=\frac{l\left(\left(n_{2}-1\right) \gamma_{i}^{\prime}+\gamma_{i}^{\prime \prime}\right)}{n_{1} n_{2}} .
\end{aligned}
$$

Из способа построения множеств $\mathcal{N}_{j}(f)$ для $j<i$ вытекает, что $V \subseteq \Theta$, поэтому

$$
\mathbf{P}_{V}\left(\mathscr{D}_{1}, \mathscr{D}_{2}, \mathscr{D}_{3}\right) \leqslant \mathbf{P}_{\Theta}\left(\mathscr{D}_{1}, \mathscr{D}_{2}, \mathscr{D}_{3}\right)=\frac{\mu_{i}}{n_{1} n_{2}} \text {. }
$$

Следовательно,

$$
l\left(\left(n_{2}-1\right) \gamma_{i}^{\prime}+\gamma_{i}^{\prime \prime}\right) \leqslant \mu_{i}
$$

Из неравенства (25) вытекает, что

$$
\mu_{h-1}=\left(1-d_{h}\right) n_{1} n_{2}<\left(n_{1}-1\right) n_{2},
$$

поэтому из неравенства (40) получаем, что

$$
m_{i+1} \leqslant \mu_{h-1}-\mu_{i}<\left(n_{1}-1\right) n_{2}-l\left(\left(n_{2}-1\right) \gamma_{i}^{\prime}+\gamma_{i}^{\prime \prime}\right)=l\left(k n_{2}-\left(n_{2}-1\right) \gamma_{i}^{\prime}-\gamma_{i}^{\prime \prime}\right) .
$$

Следовательно,

$$
m_{i+1}^{\prime \prime}<m_{i+1} / l<k n_{2}-\left(n_{2}-1\right) \gamma_{i}^{\prime}-\gamma_{i}^{\prime \prime}=\left(k-\gamma_{i}^{\prime}\right)\left(n_{2}-1\right)+k-\gamma_{i}^{\prime \prime} .
$$

Таким образом, $\hat{\alpha}_{i+1}^{\prime \prime}<k-\gamma_{i}^{\prime \prime}$. Поэтому $\gamma_{i+1}^{\prime \prime}<k$. Возьмем в качестве $\mathcal{N}_{i}(f)$ множество всех наборов $\left(\sigma_{1}, \sigma_{2}, \sigma_{3}\right)$ таких, что либо $\sigma_{2}=0$ и $\gamma_{i}^{\prime} \leqslant \sigma_{3}<k$, либо $\sigma_{2}=1$ и $\gamma_{i}^{\prime \prime} \leqslant \sigma_{3}<$ $\gamma_{i+1}^{\prime \prime}$, либо $\sigma_{3}=k$ и $\sigma_{1}=i$. Заметим, что тогда

$$
\begin{aligned}
\mathbf{P}_{\mathcal{N}_{i}(f)}\left(\mathscr{D}_{1}, \mathscr{D}_{2}, \mathscr{D}_{3}\right)= & \frac{n_{2}-1}{n_{2}}\left(\Sigma_{k}\left(\mathscr{D}_{3}\right)-\Sigma_{\gamma_{i}^{\prime}}\left(\mathscr{D}_{3}\right)\right)+\frac{1}{n_{2}}\left(\Sigma_{\gamma_{i+1}^{\prime \prime}}\left(\mathscr{D}_{3}\right)-\Sigma_{\gamma_{i}^{\prime \prime}}\left(\mathscr{D}_{3}\right)\right) \\
& \quad+\frac{1}{n_{1}} \frac{m_{i+1}^{\prime}}{n_{2}} \frac{n_{2}-1}{n_{2}}\left(\frac{l k}{n_{1}}-\frac{l \gamma_{i}^{\prime}}{n_{1}}\right)+\frac{1}{n_{2}}\left(\frac{l \gamma_{i+1}^{\prime \prime}}{n_{1}}-\frac{l \gamma_{i}^{\prime \prime}}{n_{1}}\right)+\frac{m_{i+1}^{\prime}}{n_{1} n_{2}} \\
= & \frac{\left(n_{2}-1\right) l\left(k-\gamma_{i}^{\prime}\right)+l \hat{\alpha}_{i+1}^{\prime \prime}+m_{i+1}^{\prime}}{n_{1} n_{2}}=\frac{l m_{i+1}^{\prime \prime}+m_{i+1}^{\prime}}{n_{1} n_{2}}=\frac{m_{i+1}}{n_{1} n_{2}} .
\end{aligned}
$$

2. Пусть $\gamma_{i}^{\prime} \geqslant k$. Тогда положим $\gamma_{i+1}^{\prime \prime}=\gamma_{i}^{\prime \prime}+m_{i+1}^{\prime \prime}$. Обозначим через $V$ множество наборов $\left(\sigma_{1}, \sigma_{2}, \sigma_{3}\right)$ из $\Omega$ таких, что либо $\sigma_{2}=0$ и $\sigma_{3}<k$, либо $\sigma_{2}=1$ и $\sigma_{3}<\gamma_{i}^{\prime \prime}$. Отметим, что

$$
\begin{aligned}
\mathbf{P}_{V}\left(\mathscr{D}_{1}, \mathscr{D}_{2}, \mathscr{D}_{3}\right) & =\frac{n_{2}-1}{n_{2}} \Sigma_{k}\left(\mathscr{D}_{3}\right)+\frac{1}{n_{2}} \Sigma_{\gamma_{i}^{\prime \prime}}\left(\mathscr{D}_{3}\right) \\
& =\frac{n_{2}-1}{n_{2}} \frac{l k}{n_{1}}+\frac{1}{n_{2}} \frac{l \gamma_{i}^{\prime \prime}}{n_{1}}=\frac{\left(n_{2}-1\right)\left(n_{1}-1\right)+l \gamma_{i}^{\prime \prime}}{n_{1} n_{2}} .
\end{aligned}
$$


Из способа построения множеств $\mathcal{N}_{j}(f)$ для $j<i$ вытекает, что $V \subseteq \Theta$, поэтому для $V$ справедливо соотношение (39). Следовательно,

$$
\left(n_{2}-1\right)\left(n_{1}-1\right)+l \gamma_{i}^{\prime \prime} \leqslant \mu_{i} .
$$

Поэтому, учитывая неравенство (41), получаем, что

$$
m_{i+1} \leqslant \mu_{h-1}-\mu_{i}<\left(n_{1}-1\right) n_{2}-\left(n_{2}-1\right)\left(n_{1}-1\right)-l \gamma_{i}^{\prime \prime}=l\left(k-\gamma_{i}^{\prime \prime}\right) .
$$

Следовательно, $m_{i+1}^{\prime \prime}<m_{i+1} / l<k-\gamma_{i}^{\prime \prime}$. Таким образом, $\gamma_{i+1}^{\prime \prime}<k$. Возьмем в качестве $\mathcal{N}_{i}(f)$ множество всех наборов $\left(\sigma_{1} ; \sigma_{2} ; \sigma_{3}\right)$ таких, что либо $\sigma_{2}=1$ и $\gamma_{i}^{\prime \prime} \leqslant \sigma_{3}<\gamma_{i+1}^{\prime \prime}$, либо $\sigma_{3}=k$ и $\sigma_{1}=i$. Тогда

$$
\begin{aligned}
\mathbf{P}_{\mathcal{N}_{i}(f)}\left(\mathscr{D}_{1}, \mathscr{D}_{2}, \mathscr{D}_{3}\right) & =\frac{1}{n_{2}}\left(\Sigma_{\gamma_{i+1}^{\prime \prime}}\left(\mathscr{D}_{3}\right)-\Sigma_{\gamma_{i}^{\prime \prime}}\left(\mathscr{D}_{3}\right)\right)+\frac{1}{n_{1}} \frac{m_{i+1}^{\prime}}{n_{2}} \\
& =\frac{1}{n_{2}}\left(\frac{l \gamma_{i+1}^{\prime \prime}}{n_{1}}-\frac{l \gamma_{i}^{\prime \prime}}{n_{1}}\right)+\frac{m_{i+1}^{\prime}}{n_{1} n_{2}}=\frac{l m_{i+1}^{\prime \prime}+m_{i+1}^{\prime}}{n_{1} n_{2}}=\frac{m_{i+1}}{n_{1} n_{2}} .
\end{aligned}
$$

Заметим, что для любого $i=0,1, \ldots, h-2$ в случае $\gamma_{i}^{\prime}<k$ справедливо неравенство (38) для $\gamma_{i}^{\prime \prime}$. Поэтому случай, когда $\gamma_{i}^{\prime}<k$ и $\gamma_{i}^{\prime \prime}$ не удовлетворяет неравенству (38), невозможен. Таким образом, нами рассмотрены все возможные случаи и в каждом из этих случаев построенное нами множество $\mathcal{N}_{i}(f)$ удовлетворяет соотношению (37).

Завершив построение множеств $\mathcal{N}_{i}(f)$ для $i=0,1, \ldots, h-2$, положим

$$
\mathcal{N}_{h-1}(f)=\Omega\left(\mathscr{D}_{1}, \mathscr{D}_{2}, \mathscr{D}_{3}\right) \backslash \bigcup_{i=0}^{h-2} \mathcal{N}_{i}(f) .
$$

Тогда в силу утверждения (5) из равенств (37) вытекает соотношение (36). Следовательно, поскольку $\mathscr{D}_{1}, \mathscr{D}_{2}, \mathscr{D}_{3} \in H$ и $H$ замкнуто, справедливо включение $\mathscr{D} \in H$.

Следствие 4. Пусть $p, q-\partial в а$, возможно одинаковых, простых числа, $A$ - конечное разделимое множество чисел, взаимно простых с p, b- натуральное число, взаимно простое с q, $\mathrm{H}$ - замкнутое множество стохастических векторов, содержачее множества $G[\{p\} ; A] u G[\{q\} ; b: 1]$. Тогдa $G\left[\{p\} ; \operatorname{GCD}\left(A,\{b\}^{>1}\right)\right] \subseteq H$.

Доказательство. Так как $G[\{p\} ;\|A\|: 1] \in G[\{p\} ; A]$, то $G[\{p\} ;\|A\|: 1] \subseteq H$. Из этого соотношения и соотношения $G[\{q\} ; b: 1] \subseteq H$ согласно лемме 3 следует, что $G[\{p\} ; \operatorname{gcd}(\|A\|, b): 1] \subseteq H$. Если $\operatorname{gcd}(\|A\|, b)=1$, то

$$
G[\{p\} ; \operatorname{gcd}(\|A\|, b): 1]=G[\{p\}] \supseteq G\left[\{p\} ; \operatorname{GCD}\left(A,\{b\}^{>1}\right)\right],
$$

поэтому данное утверждение в этом случае очевидно.

Пусть $\operatorname{gcd}(\|A\|, b)>1$. Из соотношений $G[\{p\} ; \operatorname{gcd}(\|A\|, b): 1] \subseteq H$ и $G[\{p\} ; A] \subseteq H$ согласно лемме 4 получаем, что $G[\{p\} ; \operatorname{GCD}(A,\{\operatorname{gcd}(\|A\|, b)\})] \subseteq H$. Для любого $a \in A$

$$
\operatorname{gcd}(a, \operatorname{gcd}(\|A\|, b))=\operatorname{gcd}(\operatorname{gcd}(a,\|A\|), b)=\operatorname{gcd}(a, b),
$$

поэтому $\operatorname{GCD}(A,\{\operatorname{gcd}(\|A\|, b)\})=\operatorname{GCD}(A,\{b\})$. Следовательно,

$$
G[\{p\} ; \operatorname{GCD}(A,\{\operatorname{gcd}(\|A\|, b)\})]=G[\{p\} ; \operatorname{GCD}(A,\{b\}\})] .
$$

Таким образом, $G\left[\{p\} ; \operatorname{GCD}\left(A,\{b\}^{>1}\right)\right]=G[\{p\} ; \operatorname{GCD}(A,\{b\})] \subseteq H$. Следствие доказано. 
Пусть $\Pi$ - множество простых чисел, $p$ - простое число, $A$ - конечное разделимое множество чисел, взаимно простых с $p$ и с множеством П. Тогда можно рассмотреть множества $G[\Pi ; A], G[\{p\} ; A], G[\Pi \cup\{p\} ; A]$ и доказать для них следующее утверждение.

Лемма 5. Пусть $H$ - замкнутое множество стохастических векторов, содержащее множества $G[\Pi ; A] u G[\{p\} ; A]$. Тогда $G[\Pi \cup\{p\} ; A] \subseteq H$.

Доказательство. Пусть $\mathscr{D}=\left(d_{1}, \ldots, d_{h}\right)-$ произвольный стохастический вектор из $G[\Pi \cup\{p\} ; A]$. Покажем, что $\mathscr{D} \in H$. Без ограничения общности мы можем рассматривать числа $d_{1}, \ldots, d_{h}$ как некоторые дроби с общим знаменателем $n_{1} n_{2}$, где $\mathscr{I}\left[n_{1}\right] \in \Pi$ и $n_{2}$ является степенью числа $p$. Положим $l=\|A\|$ и обозначим через $\hat{p}$ число $p^{\varphi(l)}$. Отметим, что согласно теореме Эйлера $\hat{p} \equiv 1(\bmod \hat{l})$. Возьмем в качестве числа $\hat{n}_{2}$ достаточно большую степень числа $\hat{p}$, не меньшую, чем $n_{2}$, и удовлетворяющую условию

$$
\hat{n}_{2}>\frac{1}{d_{h}} \text {. }
$$

Отметим, что $n_{2} \equiv 1(\bmod l)$. Выберем в качестве $\hat{n}_{1}$ достаточно большую степень числа $n_{1}$ такую, что

$$
\hat{n}_{1}>l \hat{n}_{2}(h-1) \text {. }
$$

Пусть $d_{i}=m_{i} /\left(\hat{n}_{1} \hat{n}_{2}\right)$ и $\mu_{i}=\sum_{j=1}^{i} m_{j}, i=1, \ldots, h-1$. Так как $\mathscr{D} \in G[\Pi \cup\{p\} ; A]$, для некоторых подмножеств $T_{1} \supseteq \ldots \supseteq T_{h-1}$ множества $A$ выполняются соотношения

$$
\begin{aligned}
\mu_{i} & \equiv 0 \quad\left(\bmod \left\|T_{i}\right\|\right), & i & =1, \ldots, h-1, \\
\mu_{i} & \equiv \hat{n}_{1} \hat{n}_{2} \quad\left(\bmod \left\|A \backslash T_{i}\right\|\right), & i & =1, \ldots, h-1,
\end{aligned}
$$

Для каждого $i=1, \ldots, h-1$ представим $m_{i}$ в виде $m_{i}=m_{i}^{\prime} l\left(\hat{n}_{2}-1\right)+m_{i}^{\prime \prime}$, где $m_{i}^{\prime}, m_{i}^{\prime \prime} \in \mathbf{Z}^{+}$ и $m_{i}^{\prime \prime}<l \hat{n}_{2}$. Из последнего неравенства и неравенства (43) вытекает, что

$$
\sum_{j=1}^{h-1} m_{j}^{\prime \prime}<\ln \hat{n}_{2}(h-1)<\hat{n}_{1}
$$

поэтому мы можем рассмотреть стохастический вектор

$$
\mathscr{D}^{\prime \prime}=\left(\frac{m_{1}^{\prime \prime}}{\hat{n}_{1}}, \ldots, \frac{m_{h-1}^{\prime \prime}}{\hat{n}_{1}}, \frac{1-\sum_{j=1}^{h-1} m_{j}^{\prime \prime}}{\hat{n}_{1}}\right) .
$$

Так как $\hat{n}_{1} \hat{n}_{2} \equiv \hat{n}_{1}(\bmod l)$ и все числа $\left\|A \backslash T_{i}\right\|, i=1, \ldots, h-1$, являются делителями числа $l$, из соотношений (45) следует, что

$$
\mu_{i} \equiv \hat{n}_{1} \quad\left(\bmod \left\|A \backslash T_{i}\right\|\right), \quad i=1, \ldots, h-1 .
$$

Поскольку $m_{i}^{\prime \prime} \equiv m_{i}(\bmod l)$ при $i=1, \ldots, h-1$, то

$$
\sum_{j=1}^{i} m_{i}^{\prime \prime} \equiv \mu_{i} \quad(\bmod l)
$$


и из соотношений (44) и (46) следует, что

$$
\sum_{j=1}^{i} m_{i}^{\prime \prime} \equiv 0 \quad\left(\bmod \left\|T_{i}\right\|\right), \quad \sum_{j=1}^{i} m_{i}^{\prime \prime} \equiv \hat{n}_{1} \quad\left(\bmod \left\|A \backslash T_{i}\right\|\right), \quad i=1, \ldots, h-1
$$

Таким образом, $\mathscr{D}^{\prime \prime} \in G[\Pi ; A]$, так что $\mathscr{D}^{\prime \prime} \in H$.

Отметим, что $m_{i}^{\prime} l\left(\hat{n}_{2}-1\right) \leqslant m_{i}, i=1, \ldots, h-1$, поэтому

$$
\sum_{j=1}^{h-1} m_{i}^{\prime} l\left(\hat{n}_{2}-1\right) \leqslant \mu_{h-1}
$$

Учитывая неравенство (42), получим, что

$$
\mu_{h-1}=\left(1-d_{h}\right) \hat{n}_{1} \hat{n}_{2}<\left(1-\frac{1}{\hat{n}_{2}}\right) \hat{n}_{1} \hat{n}_{2}=\hat{n}_{1}\left(\hat{n}_{2}-1\right)
$$

следовательно,

$$
\sum_{j=1}^{h-1} m_{i}^{\prime} l\left(\hat{n}_{2}-1\right)<\hat{n}_{1}\left(\hat{n}_{2}-1\right)
$$

Таким образом,

$$
\sum_{j=1}^{h-1} m_{i}^{\prime} l<\hat{n}_{1}
$$

Поэтому можно рассмотреть стохастический вектор

$$
\mathscr{D}^{\prime}=\left(\frac{m_{1}^{\prime} l}{\hat{n}_{1}}, \ldots, \frac{m_{h-1}^{\prime} l}{\hat{n}_{1}}, \frac{1-\sum_{j=1}^{h-1} m_{j}^{\prime} l}{\hat{n}_{1}}\right) .
$$

Заметим, что $\mathscr{D}^{\prime}$ принадлежит множеству $G[\Pi ; l: 1]$, которое, в свою очередь, содержится в $G[\Pi ; A]$. Таким образом, $\mathscr{D}^{\prime} \in H$.

Рассмотрим также стохастический вектор

$$
\mathscr{D}^{\prime \prime \prime}=\left(\frac{\hat{n}_{2}-1}{\hat{n}_{2}}, \frac{1}{\hat{n}_{2}}\right) \text {. }
$$

Поскольку $\hat{n}_{2}-1$ кратно числу $l$, этот вектор принадлежит множеству $G[\{p\} ; l: 1]$, содержащемуся в $G[\{p\} ; A]$. Таким образом, $\mathscr{D}^{\prime \prime \prime} \in H$.

Согласно соотношению (4), для любого $i=1, \ldots, h-1$

$$
\mathbf{P}\left\{f_{\perp}^{(h)}\left(\mathscr{D}^{\prime}, \mathscr{D}^{\prime \prime}, \mathscr{D}^{\prime \prime \prime}\right)\right\}[i]=\frac{\hat{n}_{2}-1}{\hat{n}_{2}} \frac{m_{i}^{\prime} l}{\hat{n}_{1}}+\frac{1}{\hat{n}_{2}} \frac{m_{i}^{\prime \prime}}{\hat{n}_{1}}=\frac{m_{i}}{\hat{n}_{1} \hat{n}_{2}}=d_{i} .
$$

Поэтому в силу предложения $5 \mathscr{D}=\mathbf{P}\left\{f_{\perp}^{(h)}\left(\mathscr{D}^{\prime}, \mathscr{D}^{\prime \prime}, \mathscr{D}^{\prime \prime \prime}\right)\right\}$. Поскольку $\mathscr{D}^{\prime}, \mathscr{D}^{\prime \prime}, \mathscr{D}^{\prime \prime \prime} \in H$ и $H$ замкнуто, получаем, что $\mathscr{D} \in H$.

Следствие 5. Пусть П-множество простых чисел, $A$ - конечное разделимое множество чисел, взаимно простых с множеством П, и $H$ - замкнутое множество стохастических векторов, содержащее множество $G[\{p\} ; A]$ для каждого $p$ из П. Тогда $G[\Pi ; A] \subseteq H$. 


\section{5. Основная лемма}

Пусть $p, q$ - два, возможно одинаковых, простых числа, $A$ - конечное разделимое множество чисел, взаимно простых с $p, B$ - двухэлементное разделимое множество чисел, взаимно простых с $q$. Согласно предложению 6 множество $\operatorname{GCD}(A, B)$ является разделимым и взаимно простым с $p$, поэтому мы можем рассмотреть множество стохастических векторов $G[\{p\} ; \operatorname{GCD}(A, B)]$. Доказательство нашего основного результата базируется на следующей лемме.

Лемма 6. Пусть $H$ - замкнутое множество стохастических векторов, содержащее множества $G[\{p\} ; A] u G[\{q\} ; B]$. Тогда $G[\{p\} ; \operatorname{GCD}(A, B)] \subseteq H$.

Доказательство. Пусть $B=\left\{b_{1}, b_{2}\right\}$. Положим

$$
l=\|\mathrm{GCD}(A, B)\|, \quad l^{(\sigma)}=\left\|\mathrm{GCD}\left(A,\left\{b_{\sigma}\right\}\right)\right\|, \quad \sigma=1,2 .
$$

Так как $\operatorname{gcd}\left(b_{1}, b_{2}\right)=1$, то $\operatorname{gcd}\left(l^{(1)}, l^{(2)}\right)=1$ и $l=l^{(1)} l^{(2)}$. Рассмотрим произвольный невырожденный стохастический вектор $\mathscr{D}=\left(d_{1}, \ldots, d_{h}\right)$ из $G[\{p\} ; \operatorname{GCD}(A, B)]$ с ненулевыми компонентами. Согласно предложению 2 для доказательства данной леммы достаточно показать, что $\mathscr{D} \in H$. В случае $h=2$ мы можем воспользоваться леммой 7 из [3], поэтому будем предполагать, что $h>2$. Положим $\Delta=\min _{j=1, \ldots, h} d_{j}$ и $e_{i}=\Sigma_{i}(\mathscr{D})$ для $i=1, \ldots, h-1$. Выберем достаточно большое натуральное $k$ такое, что

$$
q^{k}>2 l / d_{h}
$$

Так как $\operatorname{gcd}\left(l^{(1)}, l^{(2)}\right)=1$, согласно лемме 6 сравнение

$$
l^{(1)} x \equiv q^{k} \quad\left(\bmod l^{(2)}\right)
$$

имеет решением некоторый класс вычетов по модулю $l^{(2)}$. Обозначим через $\lambda$ наименьший положительный вычет из данного класса. Так как согласно предложению 6 множество $\operatorname{GCD}(A, B)$ взаимно просто с $q$, в силу предложения 7 выполняется равенство $\operatorname{gcd}(l, q)=1$, поэтому $\operatorname{gcd}\left(l^{(2)}, q\right)=1$. Следовательно, по крайней мере одно из чисел $\lambda$, $\lambda+l^{(2)}$ не делится на $q$. Обозначим это число через $\lambda_{1}$. Так как $\lambda_{1}$ является решением сравнения (48), существует целое $\lambda_{2}$ такое, что

$$
\lambda_{1} l^{(1)}+\lambda_{2} l^{(2)}=q^{k}
$$

Так как $\operatorname{gcd}(l, q)=1$, то $\operatorname{gcd}\left(l^{(1)}, q\right)=1$, поэтому $\operatorname{gcd}\left(\lambda_{1} l^{(1)}, q\right)=1$. Следовательно, $\operatorname{gcd}\left(\lambda_{1} l^{(1)}, \lambda_{2} l^{(2)}\right)=\operatorname{gcd}\left(\lambda_{1} l^{(1)}, q^{k}-\lambda_{1} l^{(1)}\right)=\operatorname{gcd}\left(\lambda_{1} l^{(1)}, q^{k}\right)=1$. Из способа построения числа $\lambda_{1}$ вытекает, что $0<\lambda_{1} \leqslant 2 l^{(2)}$, поэтому

$$
0<\lambda_{1} l^{(1)} \leqslant 2 l \text {. }
$$

Следовательно, учитывая соотношения (47) и (49), получаем, что $\lambda_{l} l^{(1)}<q^{k}$ и

$$
0<\lambda_{2} l^{(2)}<q^{k} \text {. }
$$

Таким образом, мы можем рассмотреть набор чисел

$$
\mathscr{D}_{2}=\left(\frac{\lambda_{1} l^{(1)}}{q^{k}}, \frac{\lambda_{2} l^{(2)}}{q^{k}}\right),
$$


который в силу равенства (49) является стохастическим вектором. Применяя предложение 8 , видим, что $l^{(\sigma)}=\operatorname{gcd}\left(\|A\|, b_{\sigma}\right), \sigma=1,2$, и поэтому

$$
\mathscr{D}_{2} \in G\left[\{q\} ; \mathrm{GCD}\left(\{\|A\|\}^{>1}, B\right)\right] \text {. }
$$

Так как $H$ содержит множество $G[\{q\} ; B]$ и подмножество $G[\{p\} ;\|A\|: 1]$ множества $G[\{p\} ; A]$, в силу следствия 4 справедливо включение $G\left[\{q\} ; \operatorname{GCD}\left(\{\|A\|\}^{>1}, B\right)\right] \subseteq H$. Следовательно, $\mathscr{D}_{2} \in H$.

Без ограничения общности мы можем рассматривать числа $e_{1}, \ldots, e_{h-1}$ как некоторые дроби с общим знаменателем $n$, являющимся степенью числа $p$. Обозначим через $n_{1}$ достаточно большую степень числа $p$, не меньшую, чем $n$, и удовлетворяющую неравенствам

$$
\begin{aligned}
& n_{1}>(h-1) q^{k} l, \\
& n_{1}>4 l^{2} / \Delta .
\end{aligned}
$$

Пусть $e_{i}=m_{i} / n_{1}, i=1, \ldots, h-1$. Так как $\mathscr{D} \in G[\{p\} ; \operatorname{GCD}(A, B)]$, существуют подмножества $T_{1}, \ldots, T_{h-1}$ множества $\operatorname{GCD}(A, B)$ такие, что справедливы соотношения

$$
\begin{array}{rlrl}
m_{i} & \equiv 0 \quad\left(\bmod \left\|T_{i}\right\|\right), & & i=1, \ldots, h-1, \\
m_{i} & \equiv n_{1} \quad\left(\bmod \left\|\operatorname{GCD}(A, B) \backslash T_{i}\right\|\right), & & i=1, \ldots, h-1, \\
T_{1} \supseteq T_{2} \supseteq \ldots \supseteq T_{h-1} . & &
\end{array}
$$

Для каждого $i=1, \ldots, h-1$ разобьем множество $A$ на подмножества

$$
\begin{aligned}
& A_{00}(i)=\left\{a \mid a \in A, \operatorname{gcd}\left(a, b_{1}\right) \notin T_{i}, \operatorname{gcd}\left(a, b_{2}\right) \notin T_{i}\right\}, \\
& A_{01}(i)=\left\{a \mid a \in A, \operatorname{gcd}\left(a, b_{1}\right) \notin T_{i}, \operatorname{gcd}\left(a, b_{2}\right) \in T_{i}\right\}, \\
& A_{10}(i)=\left\{a \mid a \in A, \operatorname{gcd}\left(a, b_{1}\right) \in T_{i}, \operatorname{gcd}\left(a, b_{2}\right) \notin T_{i}\right\}, \\
& A_{11}(i)=\left\{a \mid a \in A, \operatorname{gcd}\left(a, b_{1}\right) \in T_{i}, \operatorname{gcd}\left(a, b_{2}\right) \in T_{i}\right\} .
\end{aligned}
$$

Для $u, v=0,1, \sigma=1,2$ положим

$$
l_{u v}^{(\sigma)}(i)=\left\|\operatorname{GCD}\left(A_{u v}(i),\left\{b_{\sigma}\right\}\right)\right\|, \quad l_{u v}(i)=\left\|\operatorname{GCD}\left(A_{u v}(i), B\right)\right\| .
$$

Так как для любого фиксированного $i$ множество всех чисел $l_{u v}^{(\sigma)}(i)$ является мультипликативным разбиением множества $\operatorname{GCD}(A, B)$, согласно предложению 9 эти числа являются попарно простыми и взаимно простыми с числами $p$ и $q$. Таким образом, все числа $l_{u v}^{(\sigma)}(i)$ являются взаимно простыми с $n_{1}$ и $q$. Поскольку $\operatorname{gcd}\left(b_{1}, b_{2}\right)=1$, то $l_{u v}(i)=l_{u v}^{(1)}(i) l_{u v}^{(2)}(i)$ для любых $u, v=0,1$. Поэтому для любого фиксированного $i$ все числа $l_{u v}(i)$ также являются попарно простыми и взаимно простыми с $n_{1}$ и $q$. Кроме того, $l^{(\sigma)}=l_{00}^{(\sigma)}(i) l_{01}^{(\sigma)}(i) l_{10}^{(\sigma)}(i) l_{11}^{(\sigma)}(i)$ для $\sigma=1,2$ и, следовательно, $l=l_{00}(i) l_{01}(i) l_{10}(i) l_{11}(i)$.

Пусть $i \in\{1, \ldots, h-1\}$. Так как $\operatorname{gcd}\left(l_{01}(i) l_{11}(i), l_{00}(i) l_{10}(i)\right)=1$, согласно лемме 2 система сравнений

$$
\begin{aligned}
& x \equiv 0 \quad\left(\bmod l_{01}(i) l_{11}(i)\right) \\
& x \equiv n_{1} \quad\left(\bmod l_{00}(i) l_{10}(i)\right)
\end{aligned}
$$

имеет решение, являющееся классом вычетов по модулю $l_{01}(i) l_{11}(i) l_{00}(i) l_{10}(i)=l$. Обозначим через $v_{i}^{\prime}$ наименьший положительный вычет из данного класса. 
Аналогично, обозначим через $v_{i}^{\prime \prime}$ наименьший положительный вычет из класса вычетов по модулю $l$, являющегося решением системы сравнений

$$
\begin{aligned}
& x \equiv 0 \quad\left(\bmod l_{10}(i) l_{11}(i)\right) \\
& x \equiv n_{1} \quad\left(\bmod l_{00}(i) l_{01}(i)\right)
\end{aligned}
$$

Множество $T_{i}$ есть объединение непересекающихся множеств $\operatorname{GCD}\left(A_{10}(i),\left\{b_{1}\right\}\right)$, $\left.\operatorname{GCD}\left(A_{01}(i),\left\{b_{2}\right\}\right), \operatorname{GCD}\left(A_{11}(i),\left\{b_{1}\right\}\right\}\right)$ и $\left.\operatorname{GCD}\left(A_{11}(i),\left\{b_{2}\right\}\right\}\right)$, поэтому

$$
\left\|T_{i}\right\|=l_{10}^{(1)}(i) l_{01}^{(2)}(i) l_{11}^{(1)}(i) l_{11}^{(2)}(i) .
$$

Из соотношений (54) вытекает, что

$$
m_{i} \equiv 0 \quad\left(\bmod l_{01}^{(2)}(i) l_{11}^{(2)}(i)\right) .
$$

Множество $\operatorname{GCD}(A, B) \backslash T_{i}$ является объединением непересекающихся множеств $\left.\mathrm{GCD}\left(A_{01}(i),\left\{b_{1}\right\}\right), \operatorname{GCD}\left(A_{10}(i),\left\{b_{2}\right\}\right), \operatorname{GCD}\left(A_{00}(i),\left\{b_{1}\right\}\right\}\right)$ и $\left.\operatorname{GCD}\left(A_{00}(i),\left\{b_{2}\right\}\right\}\right)$, поэтому $\left\|\mathrm{GCD}(A, B) \backslash T_{i}\right\|=l_{01}^{(1)}(i) l_{10}^{(2)}(i) l_{00}^{(1)}(i) l_{00}^{(2)}(i)$. Из соотношений (55) вытекает, что

$$
m_{i} \equiv n_{1} \quad\left(\bmod l_{10}^{(2)}(i) l_{00}^{(2)}(i)\right) .
$$

Так как $v_{i}^{\prime}$ удовлетворяет системе (57),

$$
v_{i}^{\prime} \equiv 0 \quad\left(\bmod l_{01}(i) l_{11}(i)\right), \quad v_{i}^{\prime} \equiv n_{1} \quad\left(\bmod l_{00}(i) l_{10}(i)\right)
$$

Поэтому

$$
v_{i}^{\prime} \equiv 0 \quad\left(\bmod l_{01}^{(2)}(i) l_{11}^{(2)}(i)\right), \quad v_{i}^{\prime} \equiv n_{1} \quad\left(\bmod l_{00}^{(2)}(i) l_{10}^{(2)}(i)\right) .
$$

Сопоставляя эти два сравнения с сравнениями (59) и (60) соответственно, получаем, что $m_{i} \equiv v_{i}^{\prime}\left(\bmod l_{01}^{(2)}(i) l_{11}^{(2)}(i)\right)$ и $m_{i} \equiv v_{i}^{\prime}\left(\bmod l_{10}^{(2)}(i) l_{00}^{(2)}(i)\right)$. Таким образом, учитывая, что $\left(l_{01}^{(2)}(i) l_{11}^{(2)}(i), l_{10}^{(2)}(i) l_{00}^{(2)}(i)\right)=1$, находим, что

$$
m_{i} \equiv v_{i}^{\prime} \quad\left(\bmod l_{01}^{(2)}(i) l_{11}^{(2)}(i) l_{10}^{(2)}(i) l_{00}^{(2)}(i)\right) .
$$

Домножая обе части данного сравнения на $\lambda_{1} l^{(1)}$ и замечая, что

$$
l_{01}^{(2)}(i) l_{11}^{(2)}(i) l_{10}^{(2)}(i) l_{00}^{(2)}(i)=l^{(2)},
$$

получаем, что

$$
\lambda_{1} l^{(1)} m_{i} \equiv \lambda_{1} l^{(1)} v_{i}^{\prime}\left(\bmod l^{(2)}\right)
$$

Из равенства (49) следует, что $q^{k} \equiv \lambda_{1} l^{(1)}\left(\bmod l^{(2)}\right)$, поэтому

$$
q^{k} m_{i} \equiv \lambda_{1} l^{(1)} m_{i} \quad\left(\bmod l^{(2)}\right) .
$$

Сопоставив данное сравнение со сравнением (61), получаем соотношение

$$
q^{k} m_{i} \equiv \lambda_{1} l^{(1)} v_{i}^{\prime} \quad\left(\bmod l^{(2)}\right)
$$


Аналогичным образом получаем соотношение

$$
q^{k} m_{i} \equiv \lambda_{2} l^{(2)} v_{i}^{\prime \prime} \quad\left(\bmod l^{(1)}\right)
$$

Из соотношений (62) и (63), соответственно, вытекает, что

$$
\begin{array}{ll}
q^{k} m_{i}-\lambda_{1} l^{(1)} v_{i}^{\prime}-\lambda_{2} l^{(2)} v_{i}^{\prime \prime} \equiv 0 & \left(\bmod l^{(2)}\right), \\
q^{k} m_{i}-\lambda_{1} l^{(1)} v_{i}^{\prime}-\lambda_{2} l^{(2)} v_{i}^{\prime \prime} \equiv 0 & \left(\bmod l^{(1)}\right) .
\end{array}
$$

Следовательно, учитывая, что $\operatorname{gcd}\left(l^{(1)}, l^{(2)}\right)=1$, получаем, что

$$
q^{k} m_{i}-\lambda_{1} l^{(1)} v_{i}^{\prime}-\lambda_{2} l^{(2)} v_{i}^{\prime \prime} \equiv 0 \quad\left(\bmod l^{(1)} l^{(2)}=l\right) .
$$

Таким образом, число $\left(q^{k} m_{i}-\lambda_{1} l^{(1)} v_{i}^{\prime}-\lambda_{2} l^{(2)} v_{i}^{\prime \prime}\right) / l$ является целым. Обозначим это число через $c$. Поскольку $\operatorname{gcd}\left(\lambda_{1} l^{(1)}, \lambda_{2} l^{(2)}\right)=1$, согласно лемме 1 сравнение

$$
\lambda_{1} l^{(1)} x \equiv c \quad\left(\bmod \lambda_{2} l^{(2)}\right)
$$

имеет решением некоторый класс вычетов по модулю $\lambda_{2} l^{(2)}$. Обозначим через $\mu_{i}$ наименьший неотрица тельный вычет из данного класса. Положим

$$
x_{i}=\mu_{i}+(i-1) \lambda_{2} l^{(2)}, \quad m_{i}^{\prime}=v_{i}^{\prime}+x_{i} l .
$$

Так как $\nu_{i}^{\prime}>0$ и $\mu_{i} \geqslant 0$, то

$$
m_{i}^{\prime}>x_{i} l=(i-1) \lambda_{2} l^{(2)} l
$$

Кроме того, поскольку $v_{i}^{\prime} \leqslant l$ и $\mu_{i}<\lambda_{2} l^{(2)}$, справедливы соотношения

$$
m_{i}^{\prime} \leqslant\left(x_{i}+1\right) l=\left(\mu_{i}+1+(i-1) \lambda_{2} l^{(2)}\right) l \leqslant i \lambda_{2} l^{(2)} l .
$$

Из неравенств (65), (66) следует, что $m_{1}^{\prime}>0$ и $m_{i}<m_{i+1}$ для каждого $i=1, \ldots, h-2$. Кроме того, из неравенства (66) для $m_{h-1}^{\prime}$, учитывая соотношения (51) и (52), получаем, что

$$
m_{h-1}^{\prime} \leqslant(h-1) \lambda_{2} l^{(2)} l<(h-1) q^{k} l<n_{1} .
$$

Таким образом, справедливы неравенства

$$
0<\frac{m_{1}^{\prime}}{n_{1}}<\frac{m_{2}^{\prime}}{n_{1}}<\ldots<\frac{m_{h-1}^{\prime}}{n_{1}}<1 .
$$

Для $i=1, \ldots, h-1$ обозначим через $T_{i}^{\prime}$ подмножество $\operatorname{GCD}\left(A_{01}(i) \cup A_{11}(i),\{\|B\|\}\right)$ множества $\operatorname{GCD}(A,\{\|B\|\})$. Заметим, что

$$
\left\|T_{i}^{\prime}\right\|=\left\|\mathrm{GCD}\left(A_{01}(i),\{\|B\|\}\right)\right\|\left\|\mathrm{GCD}\left(A_{11}(i),\{\|B\|\}\right)\right\|=l_{01}(i) l_{11}(i),
$$

$\left\|\mathrm{GCD}(A,\{\|B\|\}) \backslash T_{i}^{\prime}\right\|=\left\|\mathrm{GCD}\left(A_{10}(i),\{\|B\|\}\right) t\right\|\left\|\mathrm{GCD}\left(A_{00}(i),\{\|B\|\}\right)\right\|=l_{10}(i) l_{00}(i)$.

Поскольку $m_{i}^{\prime} \equiv v_{i}^{\prime}(\bmod l)$, то $m_{i}^{\prime}$ принадлежит классу вычетов, являющемуся решением системы (57). Поэтому

$$
\begin{aligned}
& m_{i}^{\prime} \equiv 0 \quad\left(\bmod l_{01}(i) l_{11}(i)=\left\|T_{i}^{\prime}\right\|\right), \\
& m_{i}^{\prime} \equiv n_{1} \quad\left(\bmod l_{00}(i) l_{10}(i)=\left\|\mathrm{GCD}(A,\{\|B\|\}) \backslash T_{i}^{\prime}\right\|\right) .
\end{aligned}
$$


Отметим, что

$$
A_{01}(i) \cup A_{11}(i)=\left\{a \mid a \in A, \operatorname{gcd}\left(a, b_{2}\right) \in T_{i}\right\},
$$

поэтому из соотношений (56) вытекает, что

$$
A_{01}(1) \cup A_{11}(1) \supseteq A_{01}(2) \cup A_{11}(2) \supseteq \ldots \supseteq A_{01}(h-1) \cup A_{11}(h-1) .
$$

Следовательно,

$$
T_{1}^{\prime} \supseteq T_{2}^{\prime} \supseteq \ldots \supseteq T_{h-1}^{\prime}
$$

Из (67), (68), (69) следует, что стохастический вектор $\mathscr{D}_{1}^{\prime}=\left(d_{1}^{\prime}, \ldots, d_{h}^{\prime}\right)$ такой, что $\sum_{j=1}^{i} d_{j}^{\prime}=m_{i}^{\prime} / n_{1}, i=1, \ldots, h-1$, принадлежит множеству $G[\{p\} ; \operatorname{GCD}(A,\{\|B\|\})]$. Так как $H$ содержит множество $G[\{p\} ; A]$ и подмножество $G[\{q\} ;\|B\|: 1]$ множества $G[\{q\} ; B]$, согласно следствию 4 справедливо включение $G[\{p\} ;(A,\{\|B\|\})] \subseteq H$. Поэтомy $\mathscr{D}_{1}^{\prime} \in H$.

Так как $x_{i} \equiv \mu_{i}\left(\bmod \lambda_{2} l^{(2)}\right)$ для $i=1, \ldots, h-1$, то $x_{i}$ принадлежит классу вычетов, являющемуся решением сравнения (64), поэтому $\lambda_{1} l^{(1)} x_{i} \equiv c\left(\bmod \lambda_{2} l^{(2)}\right)$. Домножив модуль и обе части этого соотношения на $l$, получим, что

$$
\lambda_{1} l^{(1)} x_{i} l \equiv q^{k} m_{i}-\lambda_{1} l^{(1)} v_{i}^{\prime}-\lambda_{2} l^{(2)} v_{i}^{\prime \prime}\left(\bmod \lambda_{2} l^{(2)} l\right) .
$$

Поэтому

$$
q^{k} m_{i}-\lambda_{1} l^{(1)} m_{i}^{\prime}=q^{k} m_{i}-\lambda_{1} l^{(1)} v_{i}^{\prime}-\lambda_{1} l^{(1)} x_{i} l \equiv \lambda_{2} l^{(2)} v_{i}^{\prime \prime} \quad\left(\bmod \lambda_{2} l^{(2)} l\right) .
$$

Следовательно, число $q^{k} m_{i}-\lambda_{1} l^{(1)} m_{i}^{\prime}$ является кратным $\lambda_{2} l^{(2)}$. Обозначим целое число $\left(q^{k} m_{i}-\lambda_{1} l^{(1)} m_{i}^{\prime}\right) /\left(\lambda_{2} l^{(2)}\right)$ через $m_{i}^{\prime \prime}$. Из неравенства (66) для $m_{1}^{\prime}$, используя соотношения (50) и (51), получаем, что

$$
\lambda_{1} l^{(1)} m_{1}^{\prime} \leqslant \lambda_{1} l^{(1)} \lambda_{2} l^{(2)} l<2 l^{2} q^{k} .
$$

Применяя неравенство (53), находим, что $m_{1} \geqslant \Delta n_{1}>4 l^{2}$, поэтому, учитывая неравенство (71), получим неравенства

$$
q^{k} m_{1}-\lambda_{1} l^{(1)} m_{1}^{\prime}>4 l^{2} q^{k}-2 l^{2} q^{k}>0,
$$

так что $m_{1}^{\prime \prime}>0$. При $i=1, \ldots, h-2$ из неравенства (65) для $m_{i}^{\prime}$ и неравенства (66) для $m_{i+1}^{\prime}$ вытекает, что $m_{i+1}^{\prime}-m_{i}^{\prime}<2 \lambda_{2} l^{(2)} l$, поэтому, применяя соотношения (50) и (51), находим, что

$$
\lambda_{1} l^{(1)} m_{i+1}^{\prime}-\lambda_{1} l^{(1)} m_{i}^{\prime}<2 \lambda_{1} l^{(1)} \lambda_{2} l^{(2)} l<4 l^{2} q^{k}
$$

Заметим, что $m_{i+1}-m_{i} \geqslant \Delta n_{1}$, поэтому $m_{i+1}-m_{i}>4 l^{2}$ в силу неравенства (53). Следовательно, $q^{k} m_{i+1}-q^{k} m_{i}>4 l^{2} q^{k}$. Из этого неравенства и соотношения (72) следует, что

$$
\left(q^{k} m_{i+1}-\lambda_{1} l^{(1)} m_{i+1}^{\prime}\right)-\left(q^{k} m_{i}-\lambda_{1} l^{(1)} m_{i}^{\prime}\right)>0,
$$

поэтому $m_{i+1}^{\prime \prime}>m_{i}^{\prime \prime}$.

Согласно неравенству (47) выполняется соотношение $1-e_{h-1}=d_{h}>2 l / q^{k}$, поэтому

$$
m_{h-1}=e_{h-1} n_{1}<\left(1-2 l / q^{k}\right) n_{1} .
$$


Учитывая это неравенство, получаем, что

$$
m_{h-1}^{\prime \prime}<\frac{q^{k} m_{h-1}}{\lambda_{2} l^{(2)}}<\frac{\left(q^{k}-2 l\right) n_{1}}{\lambda_{2} l^{(2)}} .
$$

Из соотношений (49) и (50) следует, что $q^{k}-2 l \leqslant \lambda_{2} l^{(2)}$, поэтому из неравенства (73) получаем, что $m_{h-1}^{\prime \prime}<n_{1}$. Таким образом, справедливы неравенства

$$
0<\frac{m_{1}^{\prime \prime}}{n_{1}}<\frac{m_{2}^{\prime \prime}}{n_{1}}<\ldots<\frac{m_{h-1}^{\prime \prime}}{n_{1}}<1 .
$$

Для $i=1, \ldots, h-1$ обозначим через $T_{i}^{\prime \prime}$ подмножество $\operatorname{GCD}\left(A_{10}(i) \cup A_{11}(i),\{\|B\|\}\right)$ множества $\operatorname{GCD}(A,\{\|B\|\})$. Заметим, что

$$
\begin{aligned}
\left\|T_{i}^{\prime \prime}\right\| & =\left\|\mathrm{GCD}\left(A_{10}(i),\{\|B\|\}\right)\right\|\left\|\mathrm{GCD}\left(A_{11}(i),\{\|B\|\}\right)\right\|=l_{10}(i) l_{11}(i), \\
\left\|\mathrm{GCD}(A,\{\|B\|\}) \backslash T_{i}^{\prime \prime}\right\| & =\left\|\mathrm{GCD}\left(A_{01}(i),\{\|B\|\}\right)\right\|\left\|\mathrm{GCD}\left(A_{00}(i),\{\|B\|\}\right)\right\|=l_{01}(i) l_{00}(i) .
\end{aligned}
$$

Разделив обе части и модуль сравнения (70) на $\lambda_{2} l^{(2)}$, получим, что $m_{i}^{\prime \prime} \equiv v_{i}^{\prime \prime}(\bmod l)$. Следовательно, $m_{i}^{\prime \prime}$ принадлежит классу вычетов, являющемуся решением системы (58). Поэтому

$$
\begin{aligned}
& m_{i}^{\prime \prime} \equiv 0 \quad\left(\bmod l_{10}(i) l_{11}(i)=\left\|T_{i}^{\prime \prime}\right\|\right) \\
& m_{i}^{\prime \prime} \equiv n_{1} \quad\left(\bmod l_{00}(i) l_{01}(i)=\left\|\operatorname{GCD}(A,\{\|B\|\}) \backslash T_{i}^{\prime \prime}\right\|\right)
\end{aligned}
$$

Отметим, что

$$
A_{10}(i) \cup A_{11}(i)=\left\{a \mid a \in A, \operatorname{gcd}\left(a, b_{1}\right) \in T_{i}\right\},
$$

поэтому из соотношений (56) вытекает, что

$$
A_{10}(1) \cup A_{11}(1) \supseteq A_{10}(2) \cup A_{11}(2) \supseteq \ldots \supseteq A_{10}(h-1) \cup A_{11}(h-1) .
$$

Следовательно,

$$
T_{1}^{\prime \prime} \supseteq T_{2}^{\prime \prime} \supseteq \ldots \supseteq T_{h-1}^{\prime \prime}
$$

Из (74), (75), (76) получаем, что стохастический вектор $\mathscr{D}_{1}^{\prime \prime}=\left(d_{1}^{\prime \prime}, \ldots, d_{h}^{\prime \prime}\right)$ такой, что $\sum_{j=1}^{i} d_{j}^{\prime \prime}=m_{i}^{\prime \prime} / n_{1}, i=1, \ldots, h-1$, принадлежит множеству $G[\{p\} ; \operatorname{GCD}(A,\{\|B\|\})]$, содержащемуся в $H$. Поэтому $\mathscr{D}_{1}^{\prime \prime} \in H$.

Положим $\hat{\mathscr{D}}=\mathbf{P}\left\{f_{\perp}^{h}\left(\mathscr{D}_{1}^{\prime}, \mathscr{D}_{1}^{\prime \prime}, \mathscr{D}_{2}\right)\right\}$. Так как $\mathscr{D}_{1}^{\prime}, \mathscr{D}_{1}^{\prime \prime}, \mathscr{D}_{2} \in H$ и $H$ замкнуто, справедливо включение $\hat{D} \in H$. Согласно соотношению (5) для любого $i=1, \ldots, h-1$

$$
\begin{aligned}
\Sigma_{i}(\hat{D}) & =\frac{\lambda_{1} l^{(1)}}{q^{k}} \Sigma_{i}\left(\mathscr{D}_{1}^{\prime}\right)+\frac{\lambda_{2} l^{(2)}}{q^{k}} \Sigma_{i}\left(\mathscr{D}_{1}^{\prime \prime}\right)=\frac{\lambda_{1} l^{(1)}}{q^{k}} \frac{m_{i}^{\prime}}{n_{1}}+\frac{\lambda_{2} l^{(2)}}{q^{k}} \frac{m_{i}^{\prime \prime}}{n_{1}} \\
& =\frac{\lambda_{1} l^{(1)} m_{i}^{\prime}+\left(q^{k} m_{i}-\lambda_{1} l^{(1)} m_{i}^{\prime}\right)}{q^{k} n_{1}}=\frac{m_{i}}{n_{1}}=\Sigma_{i}(\mathscr{D}) .
\end{aligned}
$$

Таким образом, из предложения 3 следует, что $\mathscr{D}=\hat{\mathscr{D}}$, то есть $\mathscr{D} \in H$.

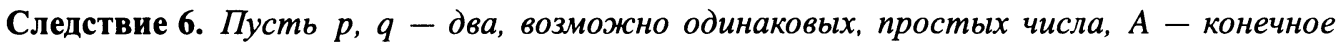
разделимое множество чисел, взаимно простых с $p, B$-взаимно простое с числом $q$ разделимое множество, состоящее из не более чем двух чисел, $H$ - замкнутое множество стохастических векторов, содержачее множества $G[\{p\} ; A] u G[\{q\} ; B]$. Тогда $G[\{p\} ; \operatorname{GCD}(A, B)] \subseteq H$. 
Доказательство. В силу леммы 6 достаточно рассмотреть случай $|B|<2$. Пусть $B=\{b\}$ - одноэлементное множество. Тогда $H$ содержит подмножество $G[\{q\} ; b: 1]$ множества $G[\{q\} ; B]$, поэтому согласно следствию 4 в $H$ содержится множество

$$
G[\{p\} ; \operatorname{GCD}(A,\{b\})]=G[\{p\} ; \operatorname{GCD}(A, B)] .
$$

Пусть теперь $B=\varnothing$. Тогда $G[\{q\} ; B]=G[\{q\}]=G[\{q\} ; 1: 1]$, поэтому согласно следствию $4 H$ содержит множество $G[\{p\} ; \operatorname{GCD}(A, \varnothing)]=G[\{p\} ; \operatorname{GCD}(A, B)]$.

Следствие 7. Пусть $p_{1}, \ldots, p_{s}$ - необязательно различные простые числа, $A_{1}, \ldots, A_{s}$ - разделимые множества, взаимно простые $c p_{1}, \ldots, p_{s}$, соответственно, состоящие из не более чем двух чисел, $H$-замкнутое множество стохастических векторов, содержащее множества $G\left[\left\{p_{i}\right\} ; A_{i}\right], i=1, \ldots, s$. Тогда $G\left[\left\{p_{1}\right\} ; \operatorname{GCD}\left(A_{1}, \ldots, A_{s}\right)\right] \subseteq H$.

\section{6. Доказательство теоремы 3}

В случае $s=1$ утверждение теоремы 3 совпадает с утверждением теоремы 2, поэтому мы можем предполагать, что $s \geqslant 2$. Пусть $\mathscr{D}=\left(d_{1}, \ldots, d_{h}\right)-$ произвольный стохастический вектор из $\langle M\rangle$. Это означает, что для некоторой $h$-значной функции $f\left(x_{1}, \ldots, x_{k}\right)$ и некоторых двумерных стохастических векторов $\mathscr{D}_{1}, \ldots, \mathscr{D}_{k}$ таких, что $\mathscr{D}_{i}[2] \in M, i=1, \ldots, k$, справедливо равенство $\mathscr{D}=\mathbf{P}\left\{f\left(\mathscr{D}_{1}, \ldots, \mathscr{D}_{k}\right)\right\}$, то есть согласно формулам (2) для каждого $i=1, \ldots, h$

$$
d_{i}=\mathbf{P}_{\mathcal{N}_{i-1}(f)}\left(\mathscr{D}_{1}, \ldots, \mathscr{D}_{k}\right)
$$

Пусть

$$
\mathscr{D}_{i}=\left(1-\frac{m_{\lambda(i)}}{n_{\lambda(i)}}, \frac{m_{\lambda(i)}}{n_{\lambda(i)}}\right), \quad i=1, \ldots, k .
$$

Положим $R=n_{\lambda(1)} \ldots n_{\lambda(k)}$ и для любого множества $U$ наборов из $\{0,1\}^{k}$ обозначим через $\mathcal{M}(U)$ число $R \mathbf{P}_{U}\left(\mathscr{D}_{1}, \ldots, \mathscr{D}_{k}\right)$. Из формулы (1) вытекает, что

$$
\mu(U)=\sum_{\left(\sigma_{1}, \ldots, \sigma_{k}\right) \in U} m_{\lambda(1)}^{\left(\sigma_{1}\right)} \ldots m_{\lambda(k)}^{\left(\sigma_{k}\right)}
$$

где $m_{j}^{(0)}=n_{j}-m_{j}$ и $m_{j}^{(1)}=m_{j}$. Таким образом, величина $M(U)$ является целым числом, и согласно равенствам (77) для каждого $i=1, \ldots, h$ число $d_{i}$ представимо дробью $M\left(\mathcal{N}_{i-1}(f)\right) / R$.

Пусть $l$ - произвольное число из $T(M)$. Тогда $l=\operatorname{gcd}\left(m_{1}^{\left(\delta_{1}\right)}, \ldots, m_{s}^{\left(\delta_{s}\right)}\right)$ для некоторых $\delta_{1}, \ldots, \delta_{s} \in\{0,1\}$. Обозначим через $\tilde{\sigma}(l)$ набор $\left(\bar{\delta}_{\lambda(1)}, \ldots, \bar{\delta}_{\lambda(k)}\right)$. Заметим, что для любого набора $\left(\sigma_{1}, \ldots, \sigma_{k}\right)$ из $\{0,1\}^{k}$, отличного от $\tilde{\sigma}(l)$, соответствующее этому набору слагаемое $m_{\lambda(1)}^{\left(\sigma_{1}\right)} \ldots m_{\lambda(k)}^{\left(\sigma_{k}\right)}$ в сумме (78) содержит сомножитель $m_{\lambda(i)}^{\left(\sigma_{i}\right)}$, делителем которого является число $l$. Поэтому величина $M(U)$ кратна числу $l$ для любого множества $U$, не содержащего набора $\tilde{\sigma}(l)$.

Для $i=1 \ldots, h-1$ обозначим через $T_{i}$ подмножество множества $T(M)$, содержащее число $l \in T(M)$ в том и только том случае, когда $i \leqslant f(\tilde{\sigma}(l))$. Пусть $l \in T_{i}$. Тогда $i \leqslant f(\tilde{\sigma}(l))$. Следовательно, любое множество $\mathcal{N}_{j}(f)$, где $j<i$, не содержит набора $\tilde{\sigma}(l)$. Поэтому в случае $j<i$ любая величина $\mathcal{M}\left(\mathcal{N}_{j}(f)\right)$ является кратной числу $l$. Тем самым сумма $\sum_{j=1}^{i} \mathcal{M}\left(\mathcal{N}_{j-1}(f)\right)$ также является кратной числу $l$. Таким образом, эта сумма 
является кратной любому числу из $T_{i}$. Так как $T_{i}$ состоит из попарно простых чисел, получаем, что

$$
\sum_{j=1}^{i} \mu\left(\mathcal{N}_{j-1}(f)\right) \equiv 0 \quad\left(\bmod \left\|T_{i}\right\|\right) .
$$

Пусть теперь $l \in T(M) \backslash T_{i}$. Это означает, что $i>f(\tilde{\sigma}(l))$. Следовательно, любое множество $\mathcal{N}_{j}(f)$, где $j \geqslant i$, не содержит набора $\tilde{\sigma}(l)$. Поэтому в случае $j \geqslant i$ любая величина $\mathcal{M}\left(\mathcal{N}_{j}(f)\right)$ является кратной числу $l$. Тем самым сумма $\sum_{j=i+1}^{h} M\left(\mathcal{N}_{j-1}(f)\right)$ также является кратной числу $l$. Таким образом, эта сумма является кратной любому числу из $T(M) \backslash T_{i}$, при этом все числа из $T(M) \backslash T_{i}$ попарно просты. Следовательно,

$$
\sum_{j=i+1}^{h} \mu\left(\mathcal{N}_{j-1}(f)\right) \equiv 0 \quad\left(\bmod \left\|T(M) \backslash T_{i}\right\|\right) .
$$

Из справедливого для стохастического вектора $\mathscr{D}$ равенства $\sum_{j=1}^{h} d_{j}=1$ вытекает, что

$$
\sum_{j=1}^{h} \mu\left(\mathcal{N}_{j-1}(f)\right)=R,
$$

поэтому из соотношения (80) получаем, что

$$
\sum_{j=1}^{i} \mathcal{M}\left(\mathcal{N}_{j-1}(f)\right) \equiv R \quad\left(\bmod \left\|T(M) \backslash T_{i}\right\|\right) .
$$

Кроме того, из определения множеств $T_{1}, \ldots, T_{h-1}$ следует, что

$$
T_{1} \supseteq T_{2} \supseteq \ldots \supseteq T_{h-1}
$$

В силу соотношений (79), (81), (82) и очевидного соотношения $\mathscr{(}(R) \subseteq \Pi(M)$ стохастический вектор

$$
\mathscr{D}=\left(\frac{\mu\left(\mathcal{N}_{0}(f)\right)}{R}, \frac{\mu\left(\mathcal{N}_{1}(f)\right)}{R}, \ldots, \frac{\mu\left(\mathcal{N}_{h-1}(f)\right)}{R}\right)
$$

принадлежит $G[\Pi(M) ; T(M)]$. Таким образом, $\langle M\rangle \subseteq G[\Pi(M) ; T(M)]$.

Докажем теперь, что для произвольного простого $p_{1}$ из П $(M)$ выполняется соотношение

$$
G\left[\left\{p_{1}\right\} ; T(M)\right] \subseteq\langle M\rangle .
$$

Без ограничения общности мы можем считать, что $p_{1}$ является делителем числа $n_{1}$. Пусть $p_{2}, \ldots, p_{s}$ - произвольные простые делители чисел $n_{2}, \ldots, n_{s}$ соответственно. Отметим, что согласно следствию 1 множество $\langle M\rangle$ является замкнутым. Согласно теореме 2 для каждого $i=1, \ldots, s$

$$
G\left[\mathscr{I}\left(n_{i}\right) ;\left\{m_{i}, n_{i}-m_{i}\right\}^{>1}\right]=\left\langle\left\{m_{i} / n_{i}\right\}\right\rangle \subseteq\langle M\rangle .
$$

Следовательно, $\langle M\rangle$ содержит подмножество $G\left[\left\{p_{i}\right\} ;\left\{m_{i}, n_{i}-m_{i}\right\}^{>1}\right]$ множества $G\left[\Phi\left(n_{i}\right) ;\left\{m_{i}, n_{i}-m_{i}\right\}^{>1}\right]$ для каждого $i=1, \ldots, s$. Поэтому, применяя следствие 7, получаем соотношение (83). Поскольку это соотношение справедливо для любого $p_{1}$ из П $(M)$, из следствия 5 вытекает, что $G[\Pi(M) ; T(M)] \subseteq\langle M\rangle$. Теорема доказана. 


\section{7. Проверка порождаемости стохастических векторов рациональными числами}

Отметим, что полученный нами результат позволяет для любого стохастического вектора $\mathscr{D}$ из SQ (множество SQ замкнуто, поэтому $\mathscr{D} \notin\langle M\rangle$ в случае $\mathscr{D} \notin \mathbf{S Q}$ ) и любого конечного множества $M$ чисел из $\mathbf{Q}(0,1)$ эффективно определить, порождается ли вектор $\mathscr{D}$ множеством $M$. Согласно теореме 3, для этого нам достаточно проверить, содержится ли вектор $\mathscr{D}$ в множестве $G[\Pi(M) ; T(M)]$.Чтобы осуществить эту проверку, сначала вычисляем множество $T(M)$. Если $M$ состоит из $s$ несократимых дробей $m_{1} / n_{1}, \ldots, m_{s} / n_{s}$, то для вычисления $T(M)$ требуется, очевидно, не более чем $O\left(2^{s}\right)$ операций взятия наибольшего общего делителя чисел, не превосходящих числа

$$
\Lambda(M)=\max \left(m_{1}, \ldots, m_{s}, n_{1}-m_{1}, \ldots, n_{s}-m_{s}\right) \text {. }
$$

Нетрудно показать, что для вычисления наибольшего общего делителя двух натуральных чисел $a, b$ с помощью алгоритма Эвклида требуется не более чем $O(\log \max (a, b))$ операций деления целых чисел. Таким образом, мы можем построить $T(M)$, используя не более чем $O\left(2^{s} \log \Lambda(M)\right)$ арифметических операций. Пусть вектор $\mathscr{D}$ имеет размерность $h$ и все его компоненты представлены несократимыми дробями. Тогда, если знаменатель какой-либо из этих компонент имеет простой делитель, не содержащийся в П $(M)$, то $\mathscr{D} \notin G[\Pi(M)]$, так что $\mathscr{D} \notin\langle M\rangle$ в силу теоремы 3. Пусть все простые делители знаменателей компонент вектора $\mathscr{D}$ содержатся в П $(M)$. Тогда для проверки принадлежности вектора $\mathscr{D}$ множеству $G[\Pi(M) ; T(M)]$ согласно сделанному нами в параграфе 3 замечанию в качестве общего знаменателя компонент вектора $\mathscr{D}$ можно взять произведение знаменателей всех его компонент, которое будем обозначать через $\eta(\mathscr{D})$. Пусть $\mu_{i}$ - сумма числителей первых $i$ компонент вектора $\mathscr{D}$, приведенных к общему знаменателю $\eta(\mathscr{D}), i=1, \ldots, h-1$. Очевидно, что для вычисления $\eta(\mathscr{D}), \mu_{1}, \ldots, \mu_{h-1}, \eta(\mathscr{D})-\mu_{1}, \ldots, \eta(\mathscr{D})-\mu_{h-1}$ достаточно выполнить не более чем $O(h)$ арифметических операций. Затем, чтобы построить соответствующие вектору $\mathscr{D}$ подмножества $T_{1} \ldots, T_{h-1}$ множества $T(M)$, для каждого числа $t$ из $T(M)$ требуется проверить делимость на $t$ чисел $\mu_{i}, \eta(\mathscr{D})-\mu_{i}$, где $i=1, \ldots, h-1$ (если $\mu_{i}$ делится на $t$, то $t \in T_{i}$, если $\eta(\mathscr{D})-\mu_{i}$ делится на $t$, то $t \in T(M) \backslash T_{i}$, если ни $\mu_{i}$, ни $\eta(\mathscr{D})-\mu_{i}$ не делятся на $t$, то заключаем, что $\left.\mathscr{D} \notin G[\Pi(M) ; T(M)]\right)$. Это требует не более чем $O(h|T(M)|)$ арифметических операций. Еще не более чем $O(h|T(M)|)$ операций требуется для проверки соотношений $T_{1} \supseteq T_{2} \supseteq \ldots \supseteq T_{h-1}$. Наконец, для того, чтобы проверить, что все простые делители знаменателей компонент вектора $\mathscr{D}$ содержатся в $\Pi(M)$, достаточно проверить соотношение

$$
\mathscr{I}(\eta(\mathscr{D})) \subseteq \mathscr{I}\left(n_{1} \ldots n_{s}\right) .
$$

Это соотношение выполняется тогда и только тогда, когда $\eta(\mathscr{D})$ является делителем достаточно большой степени числа $n_{1} \ldots n_{s}$. В качестве такой степени числа $n_{1} \ldots n_{s}$ можно, очевидно, взять любую степень, не меньшую, чем $\log _{2} \eta(D)$. В частности, такая степень может быть получена последовательным применением $\left\lceil\log _{2} \log _{2} \eta(\mathscr{D})\right\rceil$ раз к числу $n_{1} \ldots n_{s}$ операции возведения числа в квадрат, которую можно рассматривать как операцию умножения числа на само себя. Таким образом, соотношение (84) может быть проверено за не более чем $O(\log \log \eta(D))$ арифметических операций. Суммируя все этапы описанного нами алгоритма проверки соотношения $\mathscr{D} \in G[\Pi(M) ; T(M)]$, заключаем, что этот алгоритм требует не более чем $O\left(2^{s} \log \Lambda(M)+h|T(M)|+\log \log \eta(\mathscr{D})\right)$ операций. Поэтому, учитывая, что $|T(M)| \leqslant 2^{s}$, получаем следующее утверждение. 
Следствие 8. Если все элементы множества М и компоненты вектора $\mathscr{D}$ представлены несократимыми дробями, то для проверки соотночения $\mathscr{D} \in\langle M\rangle$ достаточно выполнить не более чем $O\left(2^{|M|}(\log \Lambda(M)+h)+\log \log \eta(D)\right)$ арифметических операчий.

\section{8. Заключение}

Интересным направлением дальнейших исследований является обобщение полученных результатов на случай замыканий множеств векторов из SQ произвольной размерности и на случай замыканий бесконечных множеств, что позволило бы дать описание всех замкнутых классов векторов из SQ.

Автор благодарен Ю. В. Таранникову за ценные замечания к работе.

\section{Список литературы}

1. Бухараев Р. Г., Основы теории вероятностных автоматов. Наука, Москва, 1985.

2. Виноградов И. М., Основы теории чисел. Наука, Москва, 1981.

3. Колпаков Р. М. О преобразованиях булевых случайных величин. Матем. вопросы киберн. (2000) 9, 227-252.

4. Колпаков Р. М., Замыкания одноэлементных множеств бинарных распределений с рациональными вероятностями для многозначных преобразований. Матем. вопросы киберн. (2002) 11, 63-76.

5. Колпаков Р. М., Замкнутые классы конечных распределений рациональных вероятностей. Дискретный анализ и исследование операчий (2004) 11, №3, 16-31.

6. Нурмеев Н. Н., О булевых функциях с аргументами, принимающими случайные значения. В сб.: Тезисы докл. VIII Всесоюзн. конф. «Проблемы теоретической кибернетики», т. 2. Горький, 1988 , c. 59-60.

7. Салимов Ф. И., К вопросу моделирования булевых случайных величин функциями алгебры логики. Вероятностные методы и кибернетика (1979) 15, 68-89.

8. Салимов Ф. И., Конечная порожденность некоторых алгебр над случайными величинами. Вопросы киберн. (1982) 86, 122-130.

9. Салимов Ф. И., О максимальных подалгебрах алгебр распределений. Изв. вузов. Сер. матем. (1985), №7, 14-20.

10. Салимов Ф. И., Об одном семействе алгебр распределений. Изв. вузов. Сер. матем. (1988), №7, 64-72.

11. Схиртладзе Р. Л., О синтезе $p$-схемы из контактов со случайными дискретными состояниями. Сообщ. АН Грузинской ССР (1961) 26, №2, 181-186.

12. Схиртладзе Р. Л., О методе построения булевой случайной величины с заданным распределением вероятностей. Дискретный анализ (1966) 7, 71-80.

13. Srinivasan A., Zuckerman D., Computing with very weak random sources. SIAM J. Comput. (1999) 28, №4, 1433-1459. 